\title{
Beta-Exponentiated Ishita Distribution and Its Applications
}

\author{
Samuel Ugochukwu Enogwe ${ }^{*}\left(\mathbb{D}\right.$, Gabriel Chuwukwuemeka Ibeh ${ }^{2}$ \\ ${ }^{1}$ Department of Statistics, Michael Okpara University of Agriculture, Umudike, Nigeria \\ ${ }^{2}$ Department of Mathematics/Statistics, Federal Polytechnic Nekede, Owerri, Nigeria \\ Email: *senogwe@yahoo.com, *enogwe.samuel@mouau.edu.ng
}

How to cite this paper: Enogwe, S.U. and Ibeh, G.C. (2021) Beta-Exponentiated Ishita Distribution and Its Applications. Open Journal of Statistics, 11, 690-712.

https://doi.org/10.4236/ojs.2021.115041

Received: July 31, 2021

Accepted: October 5, 2021

Published: October 8, 2021

Copyright (c) 2021 by author(s) and Scientific Research Publishing Inc. This work is licensed under the Creative Commons Attribution International License (CC BY 4.0).

http://creativecommons.org/licenses/by/4.0/

\begin{abstract}
This article develops a beta-exponentiated Ishita distribution that extends the exponentiated Ishita distribution. Expansions for the cumulative distribution and probability density functions are given. Various properties of the new distribution such as hazard function, moments, cumulants, skewness, kurtosis, mean deviations, Bonferroni and Lorenz curves, Rényi and Tsallis entropies, and stress-strength reliability are discussed. Moment generating function and characteristic function of the new model were derived. Distribution and the moment of order statistic have been derived. The method of maximum likelihood was used for estimation of parameters. The new model is quite flexible in analysing positively skewed data. Two real datasets are used to demonstrate the flexibility of the new distribution.
\end{abstract}

\section{Keywords}

Ishita Distribution, Hazard Function, Moments, Cumulants, Skewness, Kurtosis, Mean Deviation, Maximum Likelihood Estimation, Stress-Strength Reliability, Order Statistics

\section{Introduction}

The importance of probability distributions in statistical modelling cannot be overemphasized. For example, in order to build parametric analysis of variance and regression models, one is required to ensure that the research data follow certain probability distribution. Consequently, the success of many statistical methods relies on the kind of probability distribution that fits the data under consideration. For quite a number of decades, studies have been undertaken in a bid to developing probability distributions capable of modelling real life data in various fields. To this end, Shanker and Shukla [1] developed a new probability 
distribution called Ishita distribution. The cumulative distribution function (cdf) and the corresponding probability density function (pdf) of an Ishita distributed random variable $X$ having parameter $\theta$ are respectively given by

$$
F_{I}(x ; \theta)=1-\left[1+\frac{\theta x(\theta x+2)}{\theta^{3}+2}\right] \mathrm{e}^{-\theta x}
$$

and

$$
f_{I}(x ; \theta)=\frac{\theta^{3}}{\theta^{3}+2}\left(\theta+x^{2}\right) \mathrm{e}^{-\theta x}
$$

for $x>0$ and $\theta>0$. Shanker and Shukla [1] investigated the properties of the Ishita distribution and applied it to two real lifetime datasets from biomedical science and engineering. The distribution was found to outperform the exponential, Lindley and Akash distributions respectively. Another important application of the Ishita distribution is the analysis of quality control data. Al-Nasser et al. [2] developed a single-acceptance sampling plan that uses the Ishita distribution to model the lifetime distribution of a product.

It is obvious that a distribution such as Ishita distribution, which depends on one scale parameter, cannot be flexible in modelling real life data with varieties of tails. To obtain a distribution that would provide better fits to various datasets and show flexibility in statistical modelling, one either needs to add parameters to the Ishita distribution or to compound one or more distributions with Ishita distribution. Fortunately, several extensions of the Ishita distribution have been given in the literature. Notable among them are discrete Poisson-Ishita Distribution due to Shukla and Shanker [3], sized-biased Ishita distribution due to Al-Omari et al. [4], Poisson Ishita distribution proposed by Hassan et al. [5] and Transmuted Ishita distribution developed by Gharaibeh and Al-Omari [6]. Another important extension of the Ishita distribution is the Exponentiated Ishita (EI) distribution introduced by Rather and Subramanian [7] with the cumulative distribution function (cdf) given by

$$
G_{E I}(x ; \alpha, \theta)=\left[1-\left(1+\frac{\theta x(\theta x+2)}{\theta^{3}+2}\right) \mathrm{e}^{-\theta x}\right]^{\alpha}
$$

and the probability density function (pdf) corresponding to (3) is given by

$$
g_{E I}(x ; \alpha, \theta)=\frac{\alpha \theta^{3}}{\theta^{3}+2}\left(\theta+x^{2}\right)\left[1-\left(1+\frac{\theta x(\theta x+2)}{\theta^{3}+2}\right) \mathrm{e}^{-\theta x}\right]^{\alpha-1} \mathrm{e}^{-\theta x}
$$

for $x>0, \theta>0, \alpha>0$. Rather and Subramanian [7] equally studied the properties of the exponentiated Ishita (EI) distribution and demonstrated its superiority over the Ishita distribution in modelling lifetime.

In spite of the efforts made by several researchers to generalize the Ishita distribution, there is still need for more generalizations in order to obtain a new form of the Ishita distribution that will allow for the most flexible tails. To contribute in this direction, we adopt the beta-generator introduced by Eugene et al. 
[8]. Let $G(x)$ denote the cdf of the exponentiated Ishita distribution. Then the cdf of beta-exponentiated distribution is given by

$$
F(x)=I_{G(x)}(a, b)=\frac{1}{B(a, b)} \int_{0}^{G(x)} t^{a-1}(1-t)^{b-1} \mathrm{~d} t
$$

where $a>0, b>0$ are the shape parameters and $B(a, b)$ is the beta function defined by

$$
B(a, b)=\int_{0}^{1} t^{a-1}(1-t)^{b-1} \mathrm{~d} t
$$

Also, $I_{G(x)}(a, b)$ is the incomplete beta function given by

$$
I_{G(x)}(a, b)=\frac{1}{B(a, b)} \int_{0}^{G(x)} t^{a-1}(1-t)^{b-1} \mathrm{~d} t
$$

Let $f(x)$ denote the pdf of the exponentiated Ishita distribution. Then the pdf of the Beta-exponentiated distribution is given by

$$
f(x)=\frac{g(x)}{B(a, b)}[G(x)]^{a-1}[1-G(x)]^{b-1}
$$

By using (4) and (7), Eugene et al. [8] introduced a beta-normal distribution. Several other beta-generated class of distributions available in the literature are found in [9]-[38], among others.

From the existing literature on beta-generated family of distributions, one observes that the shape parameters $a$ and $b$ increases the skewness and tail weights of the Beta-G distribution, allowing for much flexibility. In view of this advantage, this article aims at introducing a four-parameter beta-exponentiated Ishita (BEI) distribution, which generalizes the exponentiated Ishita distribution. We shall derive the properties of the BEI distribution. The parameters of the new model will be estimated using maximum likelihood method. Two real life applications of the distribution will be give to illustrate the usefulness of the distribution.

\section{Beta Exponentiated Ishita Distribution}

Let $G_{E I}(x)$ denote the cdf of the exponentiated Ishita random variable $X$. Then the cdf the BEI distribution is obtained from (4) as

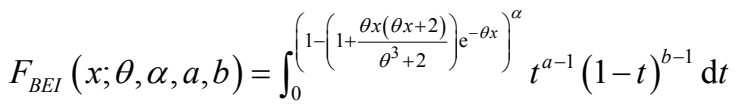

for $x>0, a>0, \theta>0, a>0, b>0$. The corresponding pdf of the BEI distribution is obtained from (7) by using (3) and (4) as

$$
\begin{aligned}
f_{B E I}(x)= & \frac{\alpha \theta^{3}\left(\theta+x^{2}\right) \mathrm{e}^{-\theta x}}{\left(\theta^{3}+2\right) B(a, b)}\left[1-\left(1-\left(1+\frac{\theta x(\theta x+2)}{\theta^{3}+2}\right) \mathrm{e}^{-\theta x}\right)^{\alpha}\right]^{b-1} \\
& \times\left(1-\left(1+\frac{\theta x(\theta x+2)}{\theta^{3}+2}\right) \mathrm{e}^{-\theta x}\right)^{\alpha a-1}
\end{aligned}
$$


for $x>0, a>0, \theta>0, a>0, b>0$. Figure 1 gives the shapes of the pdf of BEI distribution for some parameter values.

\subsection{Special Cases of the BEI Distribution}

Below are the sub-models of the BEI distribution for selected values of the parameters $\alpha, a$ and $b$.

1) When $\alpha=1$, the BEI distribution reduces to the Beta Ishita (BI) distribution, with pdf given by

$$
\begin{aligned}
f_{B I}(x ; \theta, a, b)= & \frac{\theta^{3}\left(\theta+x^{2}\right) \mathrm{e}^{-\theta x}}{\left(\theta^{3}+2\right) B(a, b)}\left(1-\left(1+\frac{\theta x(\theta x+2)}{\theta^{3}+2}\right) \mathrm{e}^{-\theta x}\right)^{a-1} \\
& \times\left[\left(1+\frac{\theta x(\theta x+2)}{\theta^{3}+2}\right) \mathrm{e}^{-\theta x}\right]^{b-1}
\end{aligned}
$$

for $x>0, \alpha>0, \theta>0, a>0, b>0$.

2) When $\alpha=a=1$, the BEI distribution reduces to another kind of Beta Ishita (BI) distribution, with pdf given by

$$
f_{1}(x ; \theta, a, b)=\frac{\theta^{3}\left(\theta+x^{2}\right) \mathrm{e}^{-\theta x}}{\left(\theta^{3}+2\right) B(1, b)}\left[\left(1+\frac{\theta x(\theta x+2)}{\theta^{3}+2}\right) \mathrm{e}^{-\theta x}\right]^{b-1} ; x>0, \theta>0, \quad b>0
$$

3) When $\alpha=b=1$, the BEI distribution reduces to a new Beta Ishita (BI) distribution, with pdf given by

$f_{2}(x)=\frac{\theta^{3}\left(\theta+x^{2}\right) \mathrm{e}^{-\theta x}}{\left(\theta^{3}+2\right) B(a, 1)}\left(1-\left(1+\frac{\theta x(\theta x+2)}{\theta^{3}+2}\right) \mathrm{e}^{-\theta x}\right)^{a-1} ; x>0, \theta>0, a>0$

4) When $\alpha=a=b=1$, the BEI distribution reduces to the Ishita (I) distribution, with pdf given by

$$
f_{I}(x ; \theta)=\frac{\theta^{3}}{\left(\theta^{3}+2\right)}\left(\theta+x^{2}\right) \mathrm{e}^{-\theta x} ; x>0, \theta>0
$$

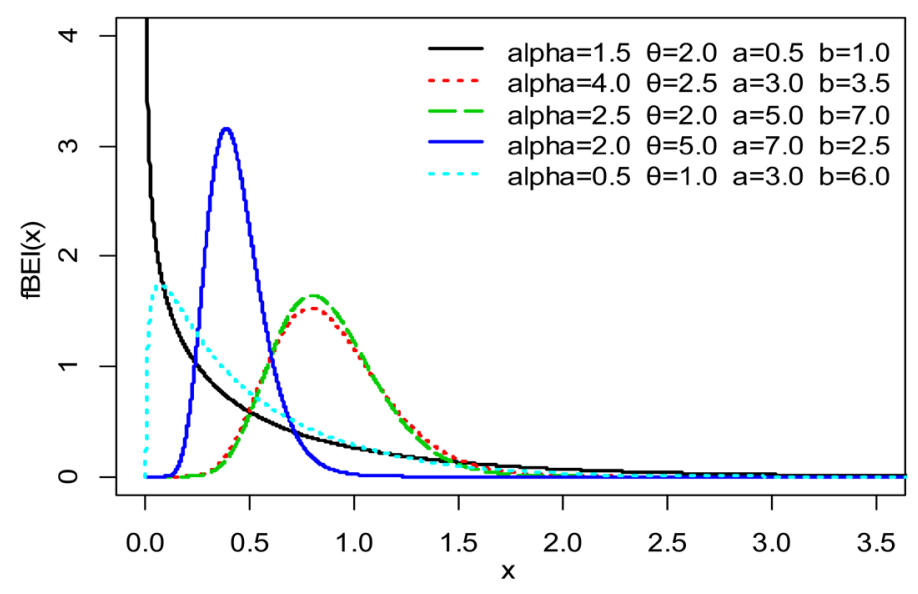

Figure 1. Various shapes of the pdf of the BEI distribution for varying values of the parameters $\alpha, \theta$, $a$ and $b$. 


\subsection{Expansion of the CDF of BEI Distribution}

The cdf of the BEI distribution in (9) appears complex and cannot be used easily for the derivation of the properties of the distribution. It is therefore necessary to provide expansion of the cdf (9) when $b>0$ is a real non-integer and when $b>0$ is an integer value.

If $\alpha(a+j)$ and $b>0$ are a real non-integer, we have the following power series expansion

$$
(1-z)^{b-1}=\sum_{j=0}^{\infty}\left(\begin{array}{c}
b-1 \\
j
\end{array}\right)(-1)^{j} z^{j}=\sum_{j=0}^{\infty} \frac{(-1)^{j} \Gamma(b) z^{j}}{\Gamma(a-j) j !}
$$

Applying the series expansion (14) on the cdf of the BEI distribution given in (8), one obtains

$$
\begin{aligned}
& F(x)=\frac{1}{B(a, b)} \sum_{j=0}^{\infty} \frac{(-1)^{j} \Gamma(b)}{\Gamma(b-j) j !} \int_{0}^{\left(1-\left(1+\frac{\theta x(\theta x+2)}{\theta^{3}+2}\right) \mathrm{e}^{-\theta x}\right)^{\alpha}} t^{a+j-1} \mathrm{~d} t \\
& =\frac{1}{B(a, b)} \sum_{j=0}^{\infty} \frac{(-1)^{j} \Gamma(b)}{(a+j) \Gamma(b-j) j !}\left(1-\left(1+\frac{\theta x(\theta x+2)}{\theta^{3}+2}\right) \mathrm{e}^{-\theta x}\right)^{\alpha(a+j)} \\
& =\frac{\alpha}{B(a, b)} \sum_{j=0}^{\infty} \frac{(-1)^{j} \Gamma(b)}{\Gamma(b-j) j !}\left\{\sum_{k=0}^{\infty} \frac{(-1)^{k} \Gamma[\alpha(a+j)]}{\Gamma[\alpha(a+j)+1-k] k !}\left(1+\frac{\theta x(\theta x+2)}{\theta^{3}+2}\right)^{k} \mathrm{e}^{-\theta k x}\right\}
\end{aligned}
$$

$F_{B E I}(x)$

$=\frac{\alpha}{B(a, b)} \sum_{j, k=0}^{\infty} \sum_{l=0}^{k} \sum_{m=0}^{l}\left(\frac{\theta^{2}}{\theta^{3}+2}\right)^{l} \frac{(-1)^{j+k}\left(\frac{2}{\theta}\right)^{m} \Gamma(b) \Gamma[\alpha(a+j)] x^{2 l-m} \mathrm{e}^{-\theta k x}}{\Gamma(b-j) \Gamma[\alpha(a+j)+1-k](k-l) !(l-m) ! j ! m !}$

If $b>0$ is an integer, then index $j$ in (14) stops at $b-1$, as a binomial expansion. If $\alpha(a+j)$ is an integer, the index $k$ stops at $\alpha(a+j)$.

\subsection{Expansion of the PDF of the BEI Distribution}

Applying the series expansion (14) on (9), we get

$$
\begin{array}{r}
f_{B E I}(x)=\frac{\alpha \theta^{3}\left(\theta+x^{2}\right) \mathrm{e}^{-\theta x}}{\left(\theta^{3}+2\right) B(a, b)} \sum_{j=0}^{\infty} \frac{(-1)^{j} \Gamma(b)}{\Gamma(b-j) j !}\left(1-\left(1+\frac{\theta x(\theta x+2)}{\theta^{3}+2}\right) \mathrm{e}^{-\theta x}\right)^{\alpha(j+a)-1} \\
=\frac{\alpha \theta^{3}\left(\theta+x^{2}\right) \mathrm{e}^{-\theta x}}{\left(\theta^{3}+2\right) B(a, b)} \sum_{j=0}^{\infty} \frac{(-1)^{j} \Gamma(b)}{\Gamma(b-j) j !} \sum_{k=0}^{\infty} \frac{(-1)^{k} \Gamma[\alpha(j+a)]}{\Gamma[\alpha(j+a)-k] k !}\left(1+\frac{\theta x(\theta x+2)}{\theta^{3}+2}\right)^{k} \mathrm{e}^{-\theta k x} \\
f_{B E I}(x)=\frac{\alpha \theta^{3}\left(\theta+x^{2}\right)}{\left(\theta^{3}+2\right) B(a, b)} \sum_{j, k=0}^{\infty} \sum_{l=0}^{k} \sum_{m=0}^{l} \frac{(-1)^{j+k}\left(\theta^{3}+2\right)^{-l} \theta^{2 l}\left(\frac{2}{\theta}\right)^{m} \Gamma(b) \Gamma[\alpha(j+a)] x^{2 l-m} \mathrm{e}^{-\theta(k+1) x}}{\Gamma(b-j) \Gamma[\alpha(j+a)-k](k-l) !(l-m) ! j ! m !}
\end{array}
$$

\subsection{Survival and Hazard Functions of the BEI Distribution}

The survival function, denoted by $S(x)=1-F(x)$, may be defined for the BEI distribution as 


$$
\begin{array}{r}
S(x)=1-\frac{\alpha}{B(a, b)} \sum_{j=0}^{\infty} \sum_{k=0}^{\infty} \sum_{l=0}^{k} \sum_{m=0}^{l}\left(\frac{\theta^{2}}{\theta^{3}+2}\right)^{l} \frac{(-1)^{j+k}\left(\frac{2}{\theta}\right)^{m} \Gamma(b) \Gamma(\alpha(a+j)) x^{2 l-m} \mathrm{e}^{-\theta k x}}{\Gamma(b-j) \Gamma(\alpha(a+j)-k+1)(k-l) !(l-m) ! j ! m !} \\
\text { The hazard function, denoted by } h(x)=f(x) / S(x), \text { may be defined for the } \\
\text { BEI distribution as } \\
h(x)=\frac{\alpha \theta^{3}\left(\theta+x^{2}\right) \mathrm{e}^{-\theta x} \sum_{j=0}^{\infty} \sum_{k=0}^{\infty} \sum_{l=0}^{k} \sum_{m=0}^{l} \frac{(-1)^{j+k}\left(\frac{\theta^{2}}{\theta^{3}+2}\right)^{l}\left(\frac{2}{\theta}\right)^{m} \Gamma(b) \Gamma(\alpha(a+j)) x^{2 l-m} \mathrm{e}^{-\theta(k+1) x}}{\Gamma(b-j) \Gamma(\alpha(a+j)-k)(k-l) !(l-m) ! j ! m !}}{\left(\theta^{3}+2\right)\left[B(a, b)-\alpha \sum_{j=0}^{\infty} \sum_{k=0}^{\infty} \sum_{l=0}^{k} \sum_{m=0}^{l}\left(\frac{\theta^{2}}{\theta^{3}+2}\right)^{l} \frac{(-1)^{j+k}\left(\frac{2}{\theta}\right)^{m} \Gamma(b) \Gamma(\alpha(a+j)) x^{2 l-m} \mathrm{e}^{-\theta k x}}{\Gamma(b-j) \Gamma(\alpha(a+j)-k+1)(k-l) !(l-m) ! j ! m !}\right]}
\end{array}
$$

Figure 2 gives the shapes of the hazard rate function for the BEI distribution for some parameter values.

\section{Properties of the BEI Distribution}

\subsection{Crude Moments of the BEI Distribution}

Let $X \sim B E I(\alpha, \theta, a, b)$. Then if $b>0$ and $\alpha(a+j)>0$ are real non-integers, the $r$ th crude moments of $X$, denoted by $\mu_{r}^{\prime}$, is given by

$$
\begin{aligned}
& \mu_{r}^{\prime}=E\left(X^{r}\right)=\int_{0}^{\infty} x^{r} f_{B E I}(x) \mathrm{d} x \\
& =\frac{\alpha \theta^{3}}{\left(\theta^{3}+2\right) B(a, b)} \sum_{j, k=0}^{\infty} \sum_{l=0}^{k} \sum_{m=0}^{l}\left(\frac{\theta^{2}}{\theta^{3}+2}\right)^{l} \frac{\left(\frac{2}{\theta}\right)^{m}(-1)^{j+k} \Gamma(b) \Gamma[\alpha(j+a)]}{\Gamma(b-j) \Gamma[\alpha(j+a)-k](k-l) !(l-m) ! j ! m !} \\
& \quad \times \int_{0}^{\infty}\left(\theta+x^{2}\right) x^{2 l-m+r} \mathrm{e}^{-\theta(k+1) x} \mathrm{~d} x
\end{aligned}
$$

Putting $y=\theta(k+1) x$ into (19) and noting that $\int_{0}^{\infty} y^{\alpha-1} \mathrm{e}^{-\theta y} \mathrm{~d} y=\Gamma(\alpha) / \theta^{\alpha}$ yields

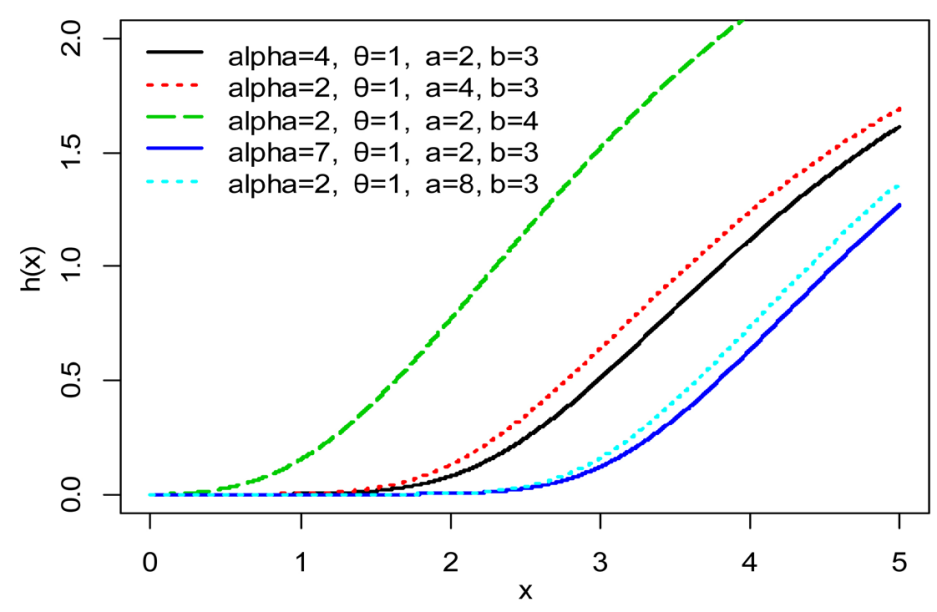

Figure 2. Plots of hazard function of the BEI distribution for selected values of the parameters $\alpha, \theta$, $a$ and $b$. 


$$
\begin{aligned}
\mu_{r}^{\prime}= & \frac{\alpha \theta^{3}}{\left(\theta^{3}+2\right) B(a, b)} \sum_{j, k=0}^{\infty} \sum_{l=0}^{k} \sum_{m=0}^{l}\left(\frac{\theta^{2}}{\theta^{3}+2}\right)^{l} \frac{\left(\frac{2}{\theta}\right)^{m}(-1)^{j+k} \Gamma(b) \Gamma[\alpha(j+a)]}{\Gamma(b-j) \Gamma[\alpha(j+a)-k](k-l) !(l-m) ! j ! m !} \\
& \times \frac{\theta^{3}(k+1)^{2} \Gamma(2 l-m+r+1)+\Gamma(2 l-m+r+3)}{[\theta(k+1)]^{2 l-m+r+3}}
\end{aligned}
$$

The first four crude moments of the BEI distribution are obtained by putting $r=1,2,3$ and 4 into (20). Thus, we obtain

$$
\begin{aligned}
& \mu_{1}^{\prime}=\frac{\alpha \theta^{3}}{\left(\theta^{3}+2\right) B(a, b)} \sum_{j, k=0}^{\infty} \sum_{l=0}^{k} \sum_{m=0}^{l}\left(\frac{\theta^{2}}{\theta^{3}+2}\right)^{l} \frac{\left(\frac{2}{\theta}\right)^{m}(-1)^{j+k} \Gamma(b) \Gamma[\alpha(j+a)]}{\Gamma(b-j) \Gamma[\alpha(j+a)-k](k-l) !(l-m) ! j ! m !} \\
& \times \frac{\theta^{3}(k+1)^{2} \Gamma(2 l-m+2)+\Gamma(2 l-m+4)}{[\theta(k+1)]^{2 l-m+4}} \\
& \mu_{2}^{\prime}=\frac{\alpha \theta^{3}}{\left(\theta^{3}+2\right) B(a, b)} \sum_{j, k=0}^{\infty} \sum_{l=0}^{k} \sum_{m=0}^{l}\left(\frac{\theta^{2}}{\theta^{3}+2}\right)^{l} \frac{\left(\frac{2}{\theta}\right)^{m}(-1)^{j+k} \Gamma(b) \Gamma[\alpha(j+a)]}{\Gamma(b-j) \Gamma[\alpha(j+a)-k](k-l) !(l-m) ! j ! m !} \\
& \times \frac{\theta^{3}(k+1)^{2} \Gamma(2 l-m+3)+\Gamma(2 l-m+5)}{[\theta(k+1)]^{2 l-m+5}} \\
& \mu_{4}^{\prime}=\frac{\alpha \theta^{3}}{\left(\theta^{3}+2\right) B(a, b)} \sum_{j, k=0}^{\infty} \sum_{l=0}^{k} \sum_{m=0}^{l}\left(\frac{\theta^{2}}{\theta^{3}+2}\right)^{l} \frac{\left(\frac{2}{\theta}\right)^{m}(-1)^{j+k} \Gamma(b) \Gamma[\alpha(j+a)]}{\Gamma(b-j) \Gamma[\alpha(j+a)-k](k-l) !(l-m) ! j ! m !} \\
& \times \frac{\theta^{3}(k+1)^{2} \Gamma(2 l-m+4)+\Gamma(2 l-m+6)}{[\theta(k+1)]^{2 l-m+6}} \\
& \mu_{4}^{\prime}=\frac{\alpha \theta^{3}}{\left(\theta^{3}+2\right) B(a, b)} \sum_{j, k=0}^{\infty} \sum_{l=0}^{k} \sum_{m=0}^{l}\left(\frac{\theta^{2}}{\theta^{3}+2}\right)^{l} \frac{\left(\frac{2}{\theta}\right)^{m}(-1)^{j+k} \Gamma(b) \Gamma[\alpha(j+a)]}{\Gamma(b-j) \Gamma[\alpha(j+a)-k](k-l) !(l-m) ! j ! m !} \\
& \times \frac{\theta^{3}(k+1)^{2} \Gamma(2 l-m+5)+\Gamma(2 l-m+7)}{[\theta(k+1)]^{2 l-m+7}}
\end{aligned}
$$

\subsection{Cumulants and Central Moments of the BEI Distribution}

The $r$ th cumulants $\left(\kappa_{r}\right)$ and $r$ th central moments $\left(\mu_{r}\right)$ of $X \sim B E I(\alpha, \theta, a, b)$ can be determined from (20) using the relations

$$
\mu_{r}=\sum_{k=0}^{r}\left(\begin{array}{l}
r \\
k
\end{array}\right)(-1)^{k}\left(\mu_{1}^{\prime}\right)^{r} \mu_{r-k}^{\prime} \quad \text { and } \kappa_{r}=\mu_{r}^{\prime}-\sum_{k=1}^{r-1}\left(\begin{array}{l}
r-1 \\
k-1
\end{array}\right) \kappa_{k} \mu_{r-k}^{\prime}
$$

Putting $r=1,2,3$ and 4 into (25), one obtains the first four cumulants of $X \sim \operatorname{BEI}(\alpha, \theta, a, b)$ as follows

$$
\begin{gathered}
\kappa_{1}=\mu_{1}^{\prime} \\
\kappa_{2}=\mu_{2}^{\prime}-\left(\mu_{1}^{\prime}\right)^{2}
\end{gathered}
$$




$$
\begin{gathered}
\kappa_{3}=\mu_{3}^{\prime}-3 \mu_{2}^{\prime} \mu_{1}^{\prime}+2\left(\mu_{1}^{\prime}\right)^{3} \\
\kappa_{4}=\mu_{4}^{\prime}-4 \mu_{3}^{\prime} \mu_{1}^{\prime}-3\left(\mu_{2}^{\prime}\right)^{2}+12 \mu_{2}^{\prime}\left(\mu_{1}^{\prime}\right)^{2}-6\left(\mu_{1}^{\prime}\right)^{4}
\end{gathered}
$$

Similarly, the following central moments of $X \sim \operatorname{BEI}(\alpha, \theta, a, b)$ are obtained from (26)-(29):

$$
\begin{gathered}
\mu_{2}=\kappa_{2} \\
\mu_{3}=\kappa_{3} \\
\mu_{4}=\kappa_{4}+3 \kappa_{2}^{2}
\end{gathered}
$$

\subsection{Skewness and Kurtosis of the BEI Distribution}

Let $X \sim \operatorname{BEI}(\alpha, \theta, a, b)$, the skewness $\left(\gamma_{1}\right)$ and Kurtosis $\left(\gamma_{2}\right)$ of $X$ can be calculated from the third and fourth standardized cumulants. Thus,

$$
\begin{aligned}
& \gamma_{1}=\frac{\kappa_{3}}{\kappa_{2}^{3 / 2}} \\
& \gamma_{2}=\frac{\kappa_{4}}{\kappa_{2}^{2}}
\end{aligned}
$$

\subsection{MGF and CF of the BEI Distribution}

Suppose $X \sim B E I(\alpha, \theta, a, b)$, then the moment generating function (mgf) of $X$ is given by

$$
\begin{aligned}
& M_{X}(t)=E\left(\mathrm{e}^{-t X}\right)=\int_{0}^{\infty} \mathrm{e}^{-t x} f_{B E I}(x) \mathrm{d} x \\
& =\frac{\alpha \theta^{3}}{\left(\theta^{3}+2\right) B(a, b)} \sum_{j, k=0}^{\infty} \sum_{l=0}^{k} \sum_{m=0}^{l}\left(\frac{\theta^{2}}{\theta^{3}+2}\right)^{l} \frac{\left(\frac{2}{\theta}\right)^{m}(-1)^{j+k} \Gamma(b) \Gamma[\alpha(j+a)]}{\Gamma(b-j) \Gamma[\alpha(j+a)-k](k-l) !(l-m) ! j ! m !} \\
& \times \int_{0}^{\infty}\left(\theta+x^{2}\right) x^{2 l-m+r} \mathrm{e}^{-[\theta(k+1)-t] x} \mathrm{~d} x
\end{aligned}
$$

Putting $y=\theta(k+1) x$ into (35) and noting that $\int_{0}^{\infty} y^{\alpha-1} \mathrm{e}^{-\theta y} \mathrm{~d} y=\Gamma(\alpha) / \theta^{\alpha}$ gives

$$
\begin{aligned}
M_{X}(t)= & \frac{\alpha \theta^{3}}{\left(\theta^{3}+2\right) B(a, b)} \sum_{j, k=0}^{\infty} \sum_{l=0}^{k} \sum_{m=0}^{l}\left(\frac{\theta^{2}}{\theta^{3}+2}\right)^{l} \frac{\left(\frac{2}{\theta}\right)^{m}(-1)^{j+k} \Gamma(b) \Gamma[\alpha(j+a)]}{\Gamma(b-j) \Gamma[\alpha(j+a)-k](k-l) !(l-m) ! j ! m !} \\
& \times \frac{\theta[\theta(k+1)-t]^{2} \Gamma(2 l-m+r+1)+\Gamma(2 l-m+r+3)}{[\theta(k+1)-t]^{2 l-m+r+3}}
\end{aligned}
$$

where $t<\theta(k+1)$. For $i=\sqrt{-1}$, the characteristic function (cf) of $X \sim B E I(\alpha, \theta, a, b)$ is given by

$$
\phi_{X}(t)=E\left(\mathrm{e}^{-i t X}\right)=\int_{0}^{\infty} \mathrm{e}^{-i t x} f_{B E I}(x) \mathrm{d} x
$$

$$
=\frac{\alpha \theta^{3}}{\left(\theta^{3}+2\right) B(a, b)} \sum_{j=0}^{\infty} \sum_{k=0}^{\infty} \sum_{l=0}^{k} \sum_{m=0}^{l}\left(\frac{\theta^{2}}{\theta^{3}+2}\right)^{l} \frac{\left(\frac{2}{\theta}\right)^{m}(-1)^{j+k} \Gamma(b) \Gamma[\alpha(j+a)]}{\Gamma(b-j) \Gamma[\alpha(j+a)-k](k-l) !(l-m) ! j ! m !}
$$




$$
\begin{aligned}
& \times \int_{0}^{\infty}\left(\theta+x^{2}\right) x^{2 l-m+r} \mathrm{e}^{-[\theta(k+1-i t)] x} \mathrm{~d} x \\
= & \frac{\alpha \theta^{3}}{\left(\theta^{3}+2\right) B(a, b)} \sum_{j=0}^{\infty} \sum_{k=0}^{\infty} \sum_{l=0}^{k} \sum_{m=0}^{l}\left(\frac{\theta^{2}}{\theta^{3}+2}\right)^{l} \frac{\left(\frac{2}{\theta}\right)^{m}(-1)^{j+k} \Gamma(b) \Gamma[\alpha(j+a)]}{\Gamma(b-j) \Gamma[\alpha(j+a)-k](k-l) !(l-m) ! j ! m !} \\
& \times \frac{\theta[\theta(k+1)-i t]^{2} \Gamma(2 l-m+r+1)+\Gamma(2 l-m+r+3)}{[\theta(k+1)-i t]^{2 l-m+r+3}}
\end{aligned}
$$

\subsection{Mean Deviations of the BEI Distribution}

The mean deviation is a special kind of the incomplete moments commonly used to measure the amount of scatter in a population. The mean deviation about the mean (when the distribution is symmetric) and the mean deviation about the median (when the distribution is skewed) are defined for $X \sim \operatorname{BEI}(\alpha, \theta, a, b)$ by

$$
M_{d}(\mu)=E(|X-\mu|)=\int_{0}^{\infty}|x-\mu| f_{B E I}(x) \mathrm{d} x
$$

and

$$
M_{d}(\tilde{\mu})=E(|X-\tilde{\mu}|)=\int_{0}^{\infty}|x-\tilde{\mu}| f_{B E I}(x) \mathrm{d} x
$$

respectively, where $\mu$ and $\tilde{\mu}$ denote, respectively the mean and median of the BEI distribution. For ease of computation, one defines $M_{d}(\mu)$ and $M_{d}(\tilde{\mu})$ respectively by

$$
M_{d}(\mu)=2 \mu F(\mu)-2 \mu+2 \int_{\mu}^{\infty} x f_{B E I}(x) \mathrm{d} x \text { and } M_{d}(\tilde{\mu})=2 \int_{\tilde{\mu}}^{\infty} x f_{B E I}(x) \mathrm{d} x-\mu
$$

To evaluate $M_{d}(\mu)$ and $M_{d}(\tilde{\mu})$, we first determine the following integrals:

$$
\begin{aligned}
& \int_{\mu}^{\infty} x f_{B E I}(x) \mathrm{d} x \\
& \left.=\frac{\alpha \theta^{3}}{\left(\theta^{3}+2\right) B(a, b)} \sum_{j, k=0}^{\infty} \sum_{l=0}^{k} \sum_{m=0}^{l} \frac{(-1)^{j+k}\left(\frac{\theta^{2}}{\theta^{3}+2}\right)^{l}\left(\frac{2}{\theta}\right)^{m} \Gamma(b) \Gamma[\alpha(j+a) \Gamma[\alpha(j+a)-k](k-l) !(l-m) ! j ! m !}{[\theta(k+1)]^{2 l-m+2}}+\frac{\Gamma(2 l-m+4, \theta(k+1) \mu)}{[\theta(k+1)]^{2 l-m+4}}\right] \\
& \times\left[\frac{\theta \Gamma(2 l-m+2, \theta(k+1) \mu)}{[\theta+\infty}\right)_{\tilde{\mu}}^{\infty} x f_{B E I}(x) \mathrm{d} x \\
& =\frac{\alpha \theta^{3}}{\left(\theta^{3}+2\right) B(a, b)} \sum_{j, k=0}^{\infty} \sum_{l=0}^{k} \sum_{m=0}^{l} \frac{(-1)^{j+k}\left(\frac{\theta^{2}}{\theta^{3}+2}\right)^{m} \Gamma(b-j) \Gamma[\alpha(j+a)-k](k-l) !(l-m) ! j ! m !}{[(\alpha+a)]} \\
& \quad \times\left[\frac{\theta \Gamma(2 l-m+2, \theta(k+1) \tilde{\mu})}{\left.[\theta(k+1)]^{2 l-m+2}+\frac{\Gamma(2 l-m+4, \theta(k+1) \tilde{\mu})}{[\theta(k+1)]^{2 l-m+4}}\right]}\right.
\end{aligned}
$$


where $\Gamma(s, x)=\int_{x}^{\infty} t^{s-1} \mathrm{e}^{-t} \mathrm{~d} t$ denote the upper incomplete gamma functions. The

expression for $\mu$ is given in (21) while the expression for $F_{B E I}(\mu)$ is deduced from (8) by replacing $x$ with $\mu$. Consequently, the mean deviations about the mean and about the median of the BEI distribution becomes

$M_{d}(\mu)=2 \mu F_{B E I}(\mu)-2 \mu+\frac{2 \alpha \theta^{3}}{\left(\theta^{3}+2\right) B(a, b)} \sum_{j, k=0}^{\infty} \sum_{l=0}^{k} \sum_{m=0}^{l} \frac{(-1)^{j+k}\left(\frac{\theta^{2}}{\theta^{3}+2}\right)^{l}\left(\frac{2}{\theta}\right)^{m} \Gamma(b) \Gamma[\alpha(j+a)]}{\Gamma(b-j) \Gamma[\alpha(j+a)-k](k-l) !(l-m) ! j ! m !} \Phi(\mu)$

and

$$
\begin{gathered}
M_{d}(\tilde{\mu})=\frac{2 \alpha \theta^{3}}{\left(\theta^{3}+2\right) B(a, b)} \sum_{j, k=0}^{\infty} \sum_{l=0}^{k} \sum_{m=0}^{l} \frac{(-1)^{j+k}\left(\frac{\theta^{2}}{\theta^{3}+2}\right)^{l}\left(\frac{2}{\theta}\right)^{m} \Gamma(b) \Gamma[\alpha(j+a)]}{\text { where } \Phi(\mu), \Phi(\tilde{\mu}) \text { and } F_{B E I}(\mu) \text { are expressed as }} \Phi(\tilde{\mu})-\mu \\
\Phi(\mu)=\left[\frac{\theta \Gamma(2 l-m+2, \theta(k+1) \mu)}{[\theta(k+1)]^{2 l-m+2}}+\frac{\Gamma(2 l-m+4, \theta(k+1) \mu)}{[\theta(k+1)]^{2 l-m+4}}\right] \\
\Phi(\tilde{\mu})=\left[\frac{\theta \Gamma(2 l-m+2, \theta(k+1) \tilde{\mu})}{\left.[\theta(k+1)]^{2 l-m+2}+\frac{\Gamma(2 l-m+4, \theta(k+1) \tilde{\mu})}{[\theta(k+1)]^{2 l-m+4}}\right]}\right. \\
F_{B E I}(\mu)=\frac{\alpha}{B(a, b)} \sum_{j, k=0}^{\infty} \sum_{l=0}^{k} \sum_{m=0}^{l}\left(\frac{\theta^{2}}{\theta^{3}+2}\right)^{l} \frac{(-1)^{j+k}\left(\frac{2}{\theta}\right)^{m} \Gamma(b) \Gamma[\alpha(a+j)] \mu^{2 l-m} \mathrm{e}^{-\theta \mu k}}{\Gamma(b-j) \Gamma[\alpha(a+j)+1-k](k-l) !(l-m) ! j ! m !}
\end{gathered}
$$

\subsection{Bonferroni and Lorenz Curves of the BEI Distribution}

The Bonferroni curve is a plot of $B(p)$ versus $q$ while the Lorenz curve is a plot of $L(p)$ versus $q$. These two curves proposed independently by Bonferroni [39] and Lorenz [40] respectively, may be defined for $X \sim B E I(\alpha, \theta, a, b)$ as

$$
B(p)=\frac{1}{p \mu} \int_{0}^{q} x f_{B E I}(x) \mathrm{d} x \text { and } L(p)=\frac{1}{\mu} \int_{0}^{q} x f_{B E I}(x) \mathrm{d} x
$$

where $\mu=E(X)$ and $q=F^{-1}(p)$. Hence,

$$
\begin{aligned}
& B(p)=\frac{\alpha \theta^{3}}{p \mu B(a, b)\left(\theta^{3}+2\right)} \sum_{j, k=0}^{\infty} \sum_{l=0}^{k} \sum_{m=0}^{l} \frac{(-1)^{j+k}\left(\frac{\theta^{2}}{\theta^{3}+2}\right)^{l}\left(\frac{2}{\theta}\right)^{m} \Gamma(b) \Gamma[\alpha(j+a)]}{\Gamma(b-j) \Gamma[\alpha(j+a)-k](k-l) !(l-m) ! j ! m !} \Psi(q) \\
& \text { and } \\
& L(p)=\frac{\alpha \theta^{3}}{\mu B(a, b)\left(\theta^{3}+2\right)} \sum_{j, k=0}^{\infty} \sum_{l=0}^{k} \sum_{m=0}^{l} \frac{(-1)^{j+k}\left(\frac{\theta^{2}}{\theta^{3}+2}\right)^{l}\left(\frac{2}{\theta}\right)^{m} \Gamma(b) \Gamma[\alpha(j+a)]}{\Gamma(b-j) \Gamma[\alpha(j+a)-k](k-l) !(l-m) ! j ! m !} \Psi(q)
\end{aligned}
$$


where

$$
\Psi(q)=\left[\frac{\theta \gamma(2 l-m+2, \theta(k+1) q)}{[\theta(k+1)]^{2 l-m+2}}+\frac{\gamma(2 l-m+4, \theta(k+1) q)}{[\theta(k+1)]^{2 l-m+4}}\right]
$$

\section{Entropy Measures of the BEI Distribution}

One of the measures of the quantity of information contained in a random sample about its parent population is the entropy. In this section, we provide the Rényi entropy proposed by Rényi [41] and the S-entropy proposed by Tsallis [42]. The Rényi entropy for $X \sim B E I(\alpha, \theta, a, b)$ is given by

$$
\begin{gathered}
E_{R}=\frac{1}{1-\gamma} \log \left(\int_{0}^{\infty} f_{B E I}^{\gamma}(x ; \alpha, \theta, a, b) \mathrm{d} x\right), \gamma>0 \text { and } \gamma \neq 1 \\
E_{R}=\frac{1}{1-\gamma} \log \left\{\left(\frac{\alpha \theta^{3}}{B(a, b)\left(\theta^{3}+2\right)}\right)^{\gamma} \int_{0}^{\infty}\left(\theta+x^{2}\right)^{\gamma} \mathrm{e}^{-\theta \gamma x}[V(x)]^{\alpha \gamma a-\gamma}\left[1-V^{\alpha}(x)\right]^{b \gamma-\gamma} \mathrm{d} x\right\}
\end{gathered}
$$

To evaluate (51), we may need the following series expansions:

$$
\begin{gathered}
\left(\theta+x^{2}\right)^{\gamma}=\sum_{h=0}^{\gamma}\left(\begin{array}{l}
\gamma \\
h
\end{array}\right) x^{2 \gamma} \theta^{\gamma-h} \\
{[V(x)]^{\alpha \gamma a-\gamma}=\left[1-\left(1+\frac{\theta x(\theta x+2)}{\theta^{3}+2}\right) \mathrm{e}^{-\theta x}\right]^{\alpha \gamma a-\gamma}} \\
=\sum_{k=0}^{\infty} \sum_{j=0}^{k} \sum_{s=0}^{j}\left(\begin{array}{c}
\alpha \gamma a-\gamma \\
k
\end{array}\right)\left(\begin{array}{l}
k \\
j
\end{array}\right)\left(\begin{array}{l}
j \\
s
\end{array}\right) \frac{(-1)^{k} 2^{s} \theta^{2 j-s} x^{2 j-s} \mathrm{e}^{-\theta k x}}{\left(\theta^{3}+2\right)^{j}} \\
{\left[1-V^{\alpha}(x)\right]^{b \gamma-\gamma}=\left[1-\left(1+\frac{\theta x(\theta x+2)}{\theta^{3}+2}\right) \mathrm{e}^{-\theta x}\right]^{\alpha \gamma a-\gamma}} \\
=\sum_{m, n=0}^{\infty} \sum_{t=0}^{n} \sum_{g=0}^{t}\left(\begin{array}{c}
b \gamma-\gamma \\
m
\end{array}\right)\left(\begin{array}{c}
\alpha m \\
n
\end{array}\right)\left(\begin{array}{l}
n \\
t
\end{array}\right)\left(\begin{array}{l}
t \\
g
\end{array}\right) \frac{(-1)^{m+n} 2^{g} \theta^{2 t-g} x^{2 t-g} \mathrm{e}^{-\theta n x}}{\left(\theta^{3}+2\right)^{t}}
\end{gathered}
$$

Substituting the expressions for $\left(\theta+x^{2}\right)^{\gamma},[V(x)]^{\alpha \gamma a-\gamma}$ and $\left[1-V^{\alpha}(x)\right]^{b \gamma-\gamma}$ into (51), yields

$$
\begin{aligned}
E_{R}= & \frac{1}{1-\gamma} \log \left\{\left(\frac{\alpha \theta^{3}}{B(a, b)\left(\theta^{3}+2\right)}\right)^{\gamma} \sum_{k, m, n=0}^{\infty} \sum_{j=0}^{k} \sum_{s=0}^{j} \sum_{t=0}^{n} \sum_{g=0}^{t} \sum_{h=0}^{\gamma}\left(\begin{array}{c}
\alpha \gamma a-\gamma \\
k
\end{array}\right)\left(\begin{array}{c}
k \\
j
\end{array}\right)\left(\begin{array}{c}
j \\
s
\end{array}\right)\left(\begin{array}{c}
b \gamma-\gamma \\
m
\end{array}\right)\left(\begin{array}{c}
\alpha m \\
n
\end{array}\right)\right. \\
& \left.\times\left(\begin{array}{l}
n \\
t
\end{array}\right)\left(\begin{array}{l}
t \\
g
\end{array}\right)\left(\begin{array}{l}
\gamma \\
h
\end{array}\right) \frac{(-1)^{k+m+n} 2^{s+g} \theta^{2(j+t)-(s+g+h)+\gamma}}{\left(\theta^{3}+2\right)^{t}} \frac{(2(j+t+\gamma)-(s+g)) !}{[\theta(\gamma+k+n)]^{2(j+t+\gamma)-(s+g)+1}}\right\}
\end{aligned}
$$

In addition, when $p=1$ and $p>0$, the s-entropy for $X \sim B E I(\alpha, \theta, a, b)$ is given by

$$
E_{S}=\frac{1}{p-1}\left[1-\int_{0}^{\infty} f_{B E I}^{p}(x ; \alpha, \theta, a, b) \mathrm{d} x\right]
$$




$$
\begin{aligned}
E_{S}= & \frac{1}{p-1}-\frac{\alpha^{p}}{(p-1)[B(a, b)]^{p}} \sum_{k, m, n=0}^{\infty} \sum_{j=0}^{k} \sum_{s=0}^{j} \sum_{t=0}^{n} \sum_{g=0}^{t} \sum_{h=0}^{\gamma}\left(\begin{array}{c}
\alpha p a-p \\
k
\end{array}\right)\left(\begin{array}{c}
k \\
j
\end{array}\right)\left(\begin{array}{c}
j \\
s
\end{array}\right)\left(\begin{array}{c}
b p-p \\
m
\end{array}\right)\left(\begin{array}{c}
\alpha m \\
n
\end{array}\right) \\
& \times\left(\begin{array}{l}
n \\
t
\end{array}\right)\left(\begin{array}{l}
t \\
g
\end{array}\right)\left(\begin{array}{l}
p \\
h
\end{array}\right) \frac{(-1)^{k+m+n} 2^{s+g} \theta^{2(j+t)-(s+g+h)+p}}{\left(\theta^{3}+2\right)^{t}} \frac{\Gamma(2(j+t+p)-(s+g)+1)}{[\theta(p+k+n)]^{2(j+t+p)-(s+g)+1}}
\end{aligned}
$$

\section{Distribution of Order Statistics}

Order statistics is very useful in reliability analysis, quality control and lifetime analysis. A better understanding of the order statistics is gotten through its distribution. In view of this, one defines the cdf of the $t$ th order statistic $X_{(r)}$ for the BEI distribution as

$$
\begin{aligned}
& F_{X_{(r)}}(x)=\sum_{i=r}^{n}\left(\begin{array}{l}
n \\
i
\end{array}\right)\left[F_{B E I}(x)\right]^{i}\left[1-F_{B E I}(x)\right]^{n-i} \\
& =\sum_{i=r}^{n} \sum_{d=0}^{n-i}\left(\begin{array}{c}
n \\
i
\end{array}\right)\left(\begin{array}{c}
n-i \\
d
\end{array}\right)\left[F_{B E I}(x)\right]^{i+d} \\
& \left.=\sum_{i=r}^{n} \sum_{d=0}^{n-i}\left(\begin{array}{c}
n \\
i
\end{array}\right)\left(\begin{array}{c}
n-i \\
d
\end{array}\right)\left[\sum_{j, k=0}^{\infty} \sum_{l=0}^{k} \sum_{m}^{l} \frac{(-1)^{j+k}\left(\theta^{3}+2\right)^{-l} \theta^{2 l}\left(\frac{2}{\theta}\right)^{m} \Gamma(b) \Gamma(\alpha(a+j)) \Gamma(b-j) \Gamma[\alpha(a+j)+1-k](k-l) !(l-m) ! j ! m !}{B(a-m}\right]^{-\theta k x}\right]^{i+d}
\end{aligned}
$$

The corresponding pdf of the $t$ th order statistic $X_{(r)}$ for the BEI distribution is given by

$$
\begin{aligned}
& f_{X_{(r)}}(x)=\frac{n !}{(r-1) !(n-r) !}\left[F_{B E I}(x)\right]^{r-1}\left[1-F_{B E I}(x)\right]^{n-r} f_{B E I}(x) \\
= & \frac{n !}{(r-1) !(n-r) !} \sum_{u=0}^{n-r}\left(\begin{array}{c}
n-r \\
u
\end{array}\right)(-1)^{u}\left[F_{B E I}(x)\right]^{r-1+u} f_{B E I}(x) \\
= & \frac{n !}{(r-1) !(n-r) !} \sum_{u=0}^{n-r}(-1)^{u}\left(\begin{array}{c}
n-r \\
u
\end{array}\right) \\
& \times\left[\sum_{j, k=0}^{\infty} \sum_{l=0}^{k} \sum_{m}^{l} \frac{(-1)^{j+k}\left(\theta^{3}+2\right)^{-l} \theta^{2 l}\left(\frac{2}{\theta}\right)^{m} \Gamma(b) \Gamma(\alpha(a+j)) x^{2 l-m} \mathrm{e}^{-\theta k x}}{B(b-j) \Gamma[\alpha(a+j)+1-k](k-l) !(l-m) ! j ! m !}\right] \\
& \times \frac{\alpha\left(\theta+x^{2}\right)}{\left(\theta^{3}+2\right)} \sum_{j=0}^{\infty} \sum_{k=0}^{\infty} \sum_{l=0}^{k} \sum_{m=0}^{l} \frac{(-1)^{j+k}\left(\theta^{3}+2\right)^{-l} \theta^{2 l+3}\left(\frac{2}{\theta}\right)^{m} \Gamma(b) \Gamma[\alpha(j+a)] x^{2 l-m} \mathrm{e}^{-\theta(k+1) x}}{B(a, b) \Gamma(b-j) \Gamma[\alpha(j+a)-k](k-l) !(l-m) ! j ! m !}
\end{aligned}
$$

The pdf of the first and nth order statistics are obtained by setting $r=1$ and $r=n$ respectively in (58).

Finally, the $q$ th moment of the $r$ th order statistic $X_{(r)}$ of the BEI distribution is given by

$$
E\left(X_{(r)}^{q}\right)=\int_{0}^{\infty} x^{q} f_{x_{(r)}}(x) \mathrm{d} x
$$




$$
\begin{aligned}
= & \frac{n !}{(r-1) !(n-r) !} \sum_{u=0}^{n-r}(-1)^{u}\left(\begin{array}{c}
n-r \\
u
\end{array}\right) \times\left[\sum_{j, k=0}^{\infty} \sum_{l=0}^{k} \sum_{m}^{l} \frac{(-1)^{j+k}\left(\theta^{3}+2\right)^{-l} \theta^{2 l}\left(\frac{2}{\theta}\right)^{m} \Gamma(b) \Gamma(\alpha(a+j))}{B(a, b) \Gamma(b-j) \Gamma[\alpha(a+j)+1-k](k-l) !(l-m) ! j ! m !}\right] \\
& \times \frac{\alpha\left(\theta+x^{2}\right)}{\left(\theta^{3}+2\right)} \sum_{j=0}^{\infty} \sum_{k=0}^{\infty} \sum_{l=0}^{k} \sum_{m=0}^{l} \frac{(-1)^{j+u}}{B(a, b) \Gamma(b-j) \Gamma[\alpha(j+a)-k](k-l) !(l-m) ! j ! m !} \\
& \times \int_{0}^{\infty} x^{2 l-m}\left(x^{2 l-m}\right)^{r-1+u}\left(\mathrm{e}^{-\theta k x}\right)^{r-l+u} \mathrm{e}^{-\theta(k+1) x} \mathrm{~d} x
\end{aligned}
$$

\section{Stress Strength Reliability}

Let $X$ be the strength of a component and $Y$, the stress applied to the component. Essentially, a component fails at the instant when the stress applied to it exceeds the strength $(Y>X)$, and the component will function satisfactorily whenever $X>Y$. Thus, a measure of component's reliability, denoted by $R=P(X>Y)$, is often required whenever a new distribution is proposed. To achieve this, we let $X \sim \operatorname{BEI}\left(\alpha_{1}, \theta_{1}, a_{1}, b_{1}\right)$ and $Y \sim \operatorname{BEI}\left(\alpha_{2}, \theta_{2}, a_{2}, b_{2}\right)$ be independent random variables. Thus,

$$
R=P(X>Y)=\int_{0}^{\infty} f_{X}\left(x ; \alpha_{1}, \theta_{1}, a_{1}, b_{1}\right) F_{Y}\left(x ; \alpha_{2}, \theta_{2}, a_{2}, b_{2}\right) \mathrm{d} x
$$

$$
\begin{aligned}
R= & \int_{0}^{\infty} \frac{\alpha_{1} \theta_{1}^{3}\left(\theta_{1}+x^{2}\right) \mathrm{e}^{-\theta_{1} x}}{B\left(a_{1}, b_{1}\right)\left(\theta_{1}^{3}+2\right)}\left(1-\left(1+\frac{\theta_{1} x\left(\theta_{1} x+2\right)}{\theta_{1}^{3}+2}\right) \mathrm{e}^{-\theta_{1} x}\right)^{\alpha_{1} a_{1}-1}\left[1-\left(1-\left(1+\frac{\theta_{1} x\left(\theta_{1} x+2\right)}{\theta_{1}^{3}+2}\right) \mathrm{e}^{-\theta_{1} x}\right)^{\alpha_{1}}\right]^{b_{1}-1} \\
& \times \frac{1}{B\left(a_{2}, b_{2}\right)\left(a_{2}+j\right)} \sum_{j=0}^{\infty}\left(\begin{array}{c}
b_{2}-1 \\
j
\end{array}\right)(-1)^{j}\left(1-\left(1+\frac{\theta_{2} x\left(\theta_{2} x+2\right)}{\theta_{2}^{3}+2}\right) \mathrm{e}^{-\theta_{2} x}\right)^{\left(a_{2}+j\right) \alpha_{2}} \mathrm{~d} x
\end{aligned}
$$

Using the binomial expansion in (60), we obtain the following

$$
\begin{aligned}
& \left(1-\left(1+\frac{\theta_{1} x\left(\theta_{1} x+2\right)}{\theta_{1}^{3}+2}\right) \mathrm{e}^{-\theta_{1} x}\right)^{\alpha_{1} a_{1}-1} \\
= & \sum_{k=0}^{\infty} \sum_{m=0}^{k} \sum_{s=0}^{m}\left(\begin{array}{c}
\alpha_{1} a_{1}-1 \\
k
\end{array}\right)\left(\begin{array}{c}
k \\
m
\end{array}\right)\left(\begin{array}{c}
m \\
s
\end{array}\right)(-1)^{k}\left(\frac{\theta_{1}^{2}}{\theta_{1}^{3}+2}\right)^{m}\left(\frac{2}{\theta_{1}}\right)^{s} x^{2 m-s} \mathrm{e}^{-\theta_{1} k x} \\
& {\left[1-\left(1-\left(1+\frac{\theta_{1} x\left(\theta_{1} x+2\right)}{\theta_{1}^{3}+2}\right) \mathrm{e}^{-\theta_{1} x}\right)^{\alpha_{1}}\right]^{b_{1}-1} } \\
= & \sum_{l=0}^{\infty} \sum_{p=0}^{\infty} \sum_{n=0}^{p} \sum_{g=0}^{n}\left(\begin{array}{c}
b_{1}-1 \\
l
\end{array}\right)\left(\begin{array}{c}
\alpha_{1} l \\
p
\end{array}\right)\left(\begin{array}{c}
p \\
m
\end{array}\right)\left(\begin{array}{l}
n \\
g
\end{array}\right)(-1)^{l+p}\left(\frac{\theta_{1}^{2}}{\theta_{1}^{3}+2}\right)^{n}\left(\frac{2}{\theta_{1}}\right)^{g} x^{2 n-g} \mathrm{e}^{-\theta_{1} p x} \\
& \left(1-\left(1+\frac{\theta_{2} x\left(\theta_{2} x+2\right)}{\theta_{2}^{3}+2}\right) \mathrm{e}^{-\theta_{2} x}\right)^{\left(a_{2}+j\right) \alpha_{2}} \\
= & \sum_{q=0}^{\infty} \sum_{t=0}^{q} \sum_{z=0}^{t}\left(\begin{array}{c}
\left(a_{2}+j\right) \alpha_{2} \\
q
\end{array}\right)\left(\begin{array}{l}
q \\
t
\end{array}\right)\left(\begin{array}{l}
t \\
z
\end{array}\right)(-1)^{q}\left(\frac{\theta_{2}^{2}}{\theta_{2}^{3}+2}\right)^{t}\left(\frac{2}{\theta_{2}}\right)^{t} x^{2 t-z} \mathrm{e}^{-\theta_{2} q x}
\end{aligned}
$$

Hence, 


$$
\begin{aligned}
& R=\int_{0}^{\infty} \frac{\alpha_{1} \theta_{1}^{3}\left(\theta_{1}+x^{2}\right) \mathrm{e}^{-\theta_{1} x}}{B\left(a_{1}, b_{1}\right)\left(\theta_{1}^{3}+2\right)} \sum_{k=0}^{\infty} \sum_{m=0}^{k} \sum_{s=0}^{m}\left(\begin{array}{c}
\alpha_{1} a_{1}-1 \\
k
\end{array}\right)\left(\begin{array}{c}
k \\
m
\end{array}\right)\left(\begin{array}{c}
m \\
s
\end{array}\right) \frac{(-1)^{k} \theta_{1}^{2 m} 2^{s} x^{2 m-s} \mathrm{e}^{-\theta_{1} k x}}{\left(\theta_{1}^{3}+2\right)^{m} \theta_{1}^{s}} \\
& \times \sum_{l=0}^{\infty} \sum_{p=0}^{\infty} \sum_{n=0}^{p} \sum_{g=0}^{n}\left(\begin{array}{c}
b_{1}-1 \\
l
\end{array}\right)\left(\begin{array}{c}
\alpha_{1} l \\
p
\end{array}\right)\left(\begin{array}{l}
p \\
m
\end{array}\right)\left(\begin{array}{l}
n \\
g
\end{array}\right) \frac{(-1)^{l+p} \theta_{1}^{2 n} 2^{g} x^{2 n-g} \mathrm{e}^{-\theta_{1} p x}}{\left(\theta_{1}^{3}+2\right)^{n} \theta_{1}^{g}} \\
& \times \frac{1}{B\left(a_{2}, b_{2}\right)\left(a_{2}+j\right)} \sum_{j=0}^{\infty}\left(\begin{array}{c}
b_{2}-1 \\
j
\end{array}\right)(-1)^{j} \sum_{q=0}^{\infty} \sum_{t=0}^{q} \sum_{z=0}^{t}\left(\begin{array}{c}
\left(a_{2}+j\right) \alpha_{2} \\
q
\end{array}\right)\left(\begin{array}{l}
q \\
t
\end{array}\right)\left(\begin{array}{l}
t \\
z
\end{array}\right) \frac{(-1)^{q} \theta_{1}^{2 t} 2^{z} x^{2 t-z} \mathrm{e}^{-\theta_{1} p x}}{\left(\theta_{2}^{3}+2\right)^{t} \theta_{2}^{t}} \mathrm{~d} x \\
& =\frac{\alpha_{1}}{B\left(a_{1}, b_{1}\right) B\left(a_{2}, b_{2}\right)} \sum_{k, l, p, j, q=0}^{\infty} \sum_{m=0}^{k} \sum_{s=0}^{m} \sum_{n=0}^{p} \sum_{g=0}^{n} \sum_{t=0}^{q} \sum_{z=0}^{t}\left(\begin{array}{c}
\alpha_{1} a_{1}-1 \\
k
\end{array}\right)\left(\begin{array}{c}
k \\
m
\end{array}\right)\left(\begin{array}{c}
m \\
s
\end{array}\right)\left(\begin{array}{c}
b_{1}-1 \\
l
\end{array}\right)\left(\begin{array}{c}
\alpha_{1} l \\
p
\end{array}\right)\left(\begin{array}{l}
p \\
m
\end{array}\right)\left(\begin{array}{l}
n \\
g
\end{array}\right) \\
& \times\left(\begin{array}{c}
b_{2}-1 \\
j
\end{array}\right)\left(\begin{array}{c}
\left(a_{2}+j\right) \alpha_{2} \\
q
\end{array}\right)\left(\begin{array}{l}
q \\
t
\end{array}\right)\left(\begin{array}{l}
t \\
z
\end{array}\right) \frac{(-1)^{k+l+p+j+q} \theta_{1}^{2(m+n+1)+1} 2^{s+g+t} \theta_{2}^{2 t}}{\left(\theta_{1}^{3}+2\right)^{m+n+1}\left(\theta_{2}^{3}+2\right)^{t} \theta_{1}^{s+g} \theta_{2}^{t}\left(a_{2}+j\right)} \\
& \times \int_{0}^{\infty}\left(\theta_{1}+x^{2}\right) x^{2(m+n+z)-(s+g+z)} \mathrm{e}^{-\left[\theta_{1}(1+k+p)+\theta_{2} q\right] x} \mathrm{~d} x \\
& R=\frac{\alpha_{1}}{B\left(a_{1}, b_{1}\right) B\left(a_{2}, b_{2}\right)} \sum_{k, l, p, j, q=0}^{\infty} \sum_{m=0}^{k} \sum_{s=0}^{m} \sum_{n=0}^{p} \sum_{g=0}^{n} \sum_{t=0}^{q} \sum_{z=0}^{t}\left(\begin{array}{c}
\alpha_{1} a_{1}-1 \\
k
\end{array}\right)\left(\begin{array}{c}
k \\
m
\end{array}\right)\left(\begin{array}{c}
m \\
s
\end{array}\right)\left(\begin{array}{c}
b_{1}-1 \\
l
\end{array}\right)\left(\begin{array}{c}
\alpha_{1} l \\
p
\end{array}\right)\left(\begin{array}{l}
p \\
m
\end{array}\right)\left(\begin{array}{l}
n \\
g
\end{array}\right) \\
& \times\left(\begin{array}{c}
b_{2}-1 \\
j
\end{array}\right)\left(\begin{array}{c}
\left(a_{2}+j\right) \alpha_{2} \\
q
\end{array}\right)\left(\begin{array}{l}
q \\
t
\end{array}\right)\left(\begin{array}{l}
t \\
z
\end{array}\right) \frac{(-1)^{k+l+p+j+q} \theta_{1}^{2(m+n+1)+1} 2^{s+g+t} \theta_{2}^{2 t}}{\left(\theta_{1}^{3}+2\right)^{m+n+1}\left(\theta_{2}^{3}+2\right)^{t} \theta_{1}^{s+g} \theta_{2}^{t}\left(a_{2}+j\right)} \\
& \times\left[\frac{\theta_{1}(2(m+n+z)-(s+g+z)) !}{\left[\theta_{1}(1+k+p)+\theta_{2} q\right]^{2(m+n+z)-(s+g+z)+1}}+\frac{(2(m+n+z)-(s+g+z)+2) !}{\left[\theta_{1}(1+k+p)+\theta_{2} q\right]^{2(m+n+z)-(s+g+z)+2}}\right]
\end{aligned}
$$

\section{Maximum Likelihood Estimation of Parameters of BEI Distribution}

Let $X_{1}, X_{2}, \cdots, X_{n}$ be a random sample of size $n$ from the BEI distribution with parameters $\alpha, \theta, a$ and $b$, then the likelihood function is given by

$$
L(\alpha, \theta, a, b)=\prod_{i=1}^{n} f_{B E I}\left(x_{i} ; \alpha, \theta, a, b\right)
$$

$$
L(\alpha, \theta, a, b)=\prod_{i=1}^{n} \frac{\alpha \theta^{3}\left(\theta+x_{i}^{2}\right) \mathrm{e}^{-\theta x_{i}}}{\left(\theta^{3}+2\right) B(a, b)}\left(1-\left(1+\frac{\theta x_{i}\left(\theta x_{i}+2\right)}{\theta^{3}+2}\right) \mathrm{e}^{-\theta x_{i}}\right)^{\alpha a-1}\left[1-\left(1-\left(1+\frac{\theta x_{i}\left(\theta x_{i}+2\right)}{\theta^{3}+2}\right) \mathrm{e}^{-\theta x_{i}}\right)^{\alpha}\right]^{b-1}
$$

The log-likelihood function is given by

$$
\begin{aligned}
\ln L(\alpha, \theta, a, b)= & n \ln (\alpha)+3 \ln (\theta)-\theta \sum_{i=1}^{n} x_{i}-n \ln \left(\theta^{3}+2\right) \\
& +n[\ln \Gamma(a, b)-\ln \Gamma(a)-\ln \Gamma(b)]+\sum_{i=1}^{n} \ln \left(\theta+x_{i}^{2}\right) \\
& +(\alpha a-1) \sum_{i=1}^{n} \ln \left(1-\left(1+\frac{\theta x_{i}\left(\theta x_{i}+2\right)}{\theta^{3}+2}\right) \mathrm{e}^{-\theta x_{i}}\right) \\
& +(b-1) \sum_{i=1}^{n} \ln \left[1-\left(1-\left(1+\frac{\theta x_{i}\left(\theta x_{i}+2\right)}{\theta^{3}+2}\right) \mathrm{e}^{-\theta x_{i}}\right)^{\alpha}\right]
\end{aligned}
$$


Differentiating (63) with respect to parameters $\alpha, \theta, a$ and $b$ and equating the resulting derivatives to zero, one obtains

$$
\begin{aligned}
\frac{\partial \ln L(\alpha, \theta, a, b)}{\partial \alpha}= & \frac{n}{\alpha}-\sum_{i=1}^{n} \frac{(b-1)\left[\theta^{3}+2-\left(\theta^{3}+2+\theta x_{i}\left(\theta x_{i}+2\right)\right) \mathrm{e}^{-\theta x_{i}}\right]^{\alpha}}{1-\left[\theta^{3}+2-\left(\theta^{3}+2+\theta x_{i}\left(\theta x_{i}+2\right)\right) \mathrm{e}^{-\theta x_{i}}\right]^{\alpha}} \\
& \times \ln \left[1-\left(1+\frac{\theta x_{i}\left(\theta x_{i}+2\right)}{\theta^{3}+2}\right) \mathrm{e}^{-\theta x_{i}}\right] \\
& +a \sum_{i=1}^{n} \ln \left[1-\left(1+\frac{\theta x_{i}\left(\theta x_{i}+2\right)}{\theta^{3}+2}\right) \mathrm{e}^{-\theta x_{i}}\right] \\
= & 0
\end{aligned}
$$

$\frac{\partial \ln L(\alpha, \theta, a, b)}{\partial \theta}$

$$
\begin{gathered}
=\frac{n}{\theta}-\frac{3 n \theta^{2}}{\theta^{3}+2}-\sum_{i=1}^{n} x_{i}+(\alpha a-1) \sum_{i=1}^{n} \frac{\left[\left(1+\frac{\theta x_{i}\left(\theta x_{i}+2\right)}{\theta^{3}+2}\right)-\left(\frac{-\theta^{4} x_{i}-4 \theta^{3}+4 \theta x_{i}+4}{\left(\theta^{3}+2\right)^{2}}\right)\right] x_{i} \mathrm{e}^{-\theta x_{i}}}{1-\left(1+\frac{\theta x_{i}\left(\theta x_{i}+2\right)}{\theta^{3}+2}\right) \mathrm{e}^{-\theta x_{i}}} \\
-\alpha(b-1) \sum_{i=1}^{n} \frac{\left[1-\left(1+\frac{\theta x_{i}\left(\theta x_{i}+2\right)}{\theta^{3}+2}\right) \mathrm{e}^{-\theta x_{i}}\right]^{\alpha-1}\left[\left(1+\frac{\theta x_{i}\left(\theta x_{i}+2\right)}{\theta^{3}+2}\right)-\left(\frac{-\theta^{4} x_{i}-4 \theta^{3}+4 \theta x_{i}+4}{\left(\theta^{3}+2\right)^{2}}\right)\right] x_{i} \mathrm{e}^{-\theta x_{i}}}{1-\left[1-\left(1+\frac{\theta x_{i}\left(\theta x_{i}+2\right)}{\theta^{3}+2}\right) \mathrm{e}^{-\theta x_{i}}\right]^{\alpha}}
\end{gathered}
$$

$=0$

$$
\begin{aligned}
& \frac{\partial \ln L(\alpha, \theta, a, b)}{\partial a} \\
= & n[\psi(a+b)-\psi(a)]+\alpha \sum_{i=1}^{n} \ln \left(1-\left(1+\frac{\theta x_{i}\left(\theta x_{i}+2\right)}{\theta^{3}+2}\right) \mathrm{e}^{-\theta x_{i}}\right) \\
\quad= & 0 \\
\frac{\partial \ln L(\alpha, \theta, a, b)}{\partial b} & n[\psi(a+b)-\psi(b)]+\sum_{i=1}^{n} \ln \left[1-\left(1-\left(1+\frac{\theta x_{i}\left(\theta x_{i}+2\right)}{\theta^{3}+2}\right) \mathrm{e}^{-\theta x_{i}}\right)^{\alpha}\right] \\
= & 0
\end{aligned}
$$

where $\psi(x)=\mathrm{d} \log \Gamma(x) / \mathrm{d} x$ is the digamma function. It may be observed that the log-likelihood equations do not admit any explicit solution for the maximum likelihood estimates (MLEs) of the BEI distribution due its non-linear structure. Therefore, the MLEs of the parameters of the BEI distribution, denoted by $\hat{\Theta}=(\hat{\alpha}, \hat{\theta}, \hat{a}, \hat{b})^{\mathrm{T}}$ are obtained using the Newton-Raphson's numerical approach with the aid of R software. 


\section{Asymptotic Confidence Intervals of the Parameters of BEI Distribution}

Let $\hat{\Theta}=(\hat{\alpha}, \hat{\theta}, \hat{a}, \hat{b})^{\mathrm{T}}$ be the MLE of $\Theta=(\alpha, \theta, a, b)^{\mathrm{T}}$ for the BEI distribution. To construct the confidence intervals, we need the Fisher information matrix, denoted by $I(\Theta)$. Thus,

$$
I(\Theta)=\left(\begin{array}{cccc}
I_{\alpha \alpha} & I_{\alpha \theta} & I_{\alpha a} & I_{\alpha b} \\
I_{\alpha \theta} & I_{\theta \theta} & I_{a \theta} & I_{b \theta} \\
I_{\alpha a} & I_{a \theta} & I_{a a} & I_{a b} \\
I_{\alpha b} & I_{b \theta} & I_{a b} & I_{b b}
\end{array}\right)
$$

The elements of (68) are the second derivatives of (68) with respect to the parameters of the BEI distribution. As pointed out in Lehmann and Casella [43], the asymptotic distribution of $\sqrt{n}(\hat{\Theta}-\Theta)$ is $N_{4}\left(\mathbf{0}, \boldsymbol{I}^{-1}(\Theta)\right)$, under certain regularity conditions. Consequently, the approximate $100(1-\tau) \%$ two-sided confidence intervals for $\alpha, \theta, a$ and $b$ are given, respectively, by

$\hat{\alpha} \pm Z_{\tau / 2} \sqrt{I_{\alpha \alpha}^{-1}(\hat{\Theta})}, \quad \hat{\theta} \pm Z_{\tau / 2} \sqrt{I_{\theta \theta}^{-1}(\hat{\Theta})}, \quad \hat{a} \pm Z_{\tau / 2} \sqrt{I_{a a}^{-1}(\hat{\Theta})}$ and $\hat{b} \pm Z_{\tau / 2} \sqrt{I_{b b}^{-1}(\hat{\Theta})}$

where $I_{\alpha \alpha}^{-1}(\hat{\Theta}), I_{\theta \theta}^{-1}(\hat{\Theta}), I_{a a}^{-1}(\hat{\Theta})$ and $I_{b b}^{-1}(\hat{\Theta})$ are the diagonal elements of the matrix $\boldsymbol{I}_{n}^{-1}(\hat{\Theta})$ and $Z_{\tau / 2}$ is the upper $(\tau / 2)$ th percentile of a standard normal distribution.

\section{Applications}

The performance of the BEI vis-à-vis other related distributions considered in this study in fitting two real data sets is demonstrated in this section. The estimation of the parameters of each of the distributions is made using the maximum likelihood method of estimation. The standard error of each of the parameters estimated is enclosed in brackets. The performance measures obtained include Log-likelihood (LL), Akaike's information criterion (AIC), Kolmogorov-smirnov (K-S) statistic, and the corresponding probability value ( $\mathrm{p}$-value). The comparison of the proposed distribution is conducted with some well-known lifetime distributions such as the Ishita distribution (ID), Exponentiated Ishita distribution (EID), Akash distribution (AD), Exponentiated Akash distribution (EAD), Exponential distribution (ED) and Exponentiated Exponential distribution (EED) respectively.

\section{Dataset one}

The first data represents the sum of skin folds in 202 athletes collected at the Australian Institute of sports and used in a book by Weisberg [44]. The data is given below:

28.0, 98.0, 89.0, 68.9, 69.9, 109.0, 52.3, 52.8, 46.7, 82.7, 42.3, 109.1, 96.8, 98.3, 103.6, 110.2, 98.1, 57.0, 43.1, 71.1, 29.7, 96.3, 102.8, 80.3, 122.1, 71.3, 200.8, 80.6, $65.3,78.0,65.9,38.9,56.5,104.6,74.9,90.4,54.6,131.9,68.3,52.0,40.8,34.3$, $44.8,105.7,126.4,83.0,106.9,88.2,33.8,47.6,42.7,41.5,34.6,30.9,100.7,80.3$, 
$91.0,156.6,95.4,43.5,61.9,35.2,50.9,31.8,44.0,56.8,75.2,76.2,101.1,47.5$, $46.2,38.2,49.2,49.6,34.5,37.5,75.9,87.2,52.6,126.4,55.6,73.9,43.5,61.8,88.9$, 31.0, 37.6, 52.8, 97.9, 111.1, 114.0, 62.9, 36.8, 56.8, 46.5, 48.3, 32.6, 31.7, 47.8, $75.1,110.7,70.0,52.5,67.0,41.6,34.8,61.8,31.5,36.6,76.0,65.1,74.7,77.0,62.6$, $41.1,58.9,60.2,43.0,32.6,48.0,61.2,171.1,113.5,148.9,49.9,59.4,44.5,48.1$, 61.1, 31.0, 41.9, 75.6, 76.8, 99.8, 80.1, 57.9, 48.4, 41.8, 44.5, 43.8, 33.7, 30.9, 43.3, $117.8,80.3,156.6,109.6,50.0,33.7,54.0,54.2,30.3,52.8,49.5,90.2,109.5,115.9$, $98.5,54.6,50.9,44.7,41.8,38.0,43.2,70.0,97.2,123.6,181.7,136.3,42.3,40.5$, 64.9, 34.1, 55.7, 113.5, 75.7, 99.9, 91.2, 71.6, 103.6, 46.1, 51.2, 43.8, 30.5, 37.5, $96.9,57.7,125.9,49.0,143.5,102.8,46.3,54.4,58.3,34.0,112.5,49.3,67.2,56.5$, $47.6,60.4,34.9$

Using (63), (68), and (69), we obtain the values reported in Table 1, which are the estimated parameters, their standard errors and confidence intervals for data set 1 . Table 2 gives the Log-likelihood values, K-S statistic and their p-values, AIC and BIC values for data set 1 .

\section{Dataset two}

The second data set represents time of failure $\left(10^{3} \mathrm{~h}\right)$ of turbocharger of one type of engine (Xu et al., [45]):

$1.6,3.5,4.8,5.4,6.0,6.5,7.0,7.3,7.7,8.0,8.4,2.0,3.9,5.0,5.6,6.1,6.5,7.1,7.3,7.8$, 8.1, 8.4, 2.6, 4.5, 5.1, 5.8, 6.3, 6.7, 7.3, 7.7, 7.9, 8.3, 8.5, 3.0, 4.6, 5.3, 6.0, 8.7, 8.8, 9.0

Table 1. The MLEs of the parameters of the fitted distributions and their confidence intervals.

\begin{tabular}{|c|c|c|c|c|c|}
\hline Distribution & Parameter & MLE & Standard Error & Lower Bound & Upper Bound \\
\hline & $\alpha$ & 6.0540 & 0.0582 & 5.93993 & 6.1681 \\
\hline \multirow[t]{3}{*}{ BEID } & $\theta$ & 0.2125 & 0.0026 & 0.20740 & 0.2176 \\
\hline & $a$ & 4.0831 & 0.7428 & 2.62721 & 5.5390 \\
\hline & $b$ & 0.1493 & 0.0115 & 0.12676 & 0.1718 \\
\hline ID & $\theta$ & 0.0435 & 0.0018 & 0.03997 & 0.0470 \\
\hline \multirow[t]{2}{*}{ EID } & $\alpha$ & 0.6210 & 0.0417 & 0.53927 & 0.7027 \\
\hline & $\theta$ & 0.0351 & 0.0021 & 0.03098 & 0.0392 \\
\hline $\mathrm{AD}$ & $\theta$ & 0.0434 & 0.0018 & 0.03987 & 0.0469 \\
\hline \multirow[t]{2}{*}{ EAD } & $\alpha$ & 2.1150 & 0.2815 & 1.56326 & 2.6667 \\
\hline & $\theta$ & 0.0584 & 0.0034 & 0.05174 & 0.0651 \\
\hline $\mathrm{LD}$ & $\theta$ & 0.0286 & 0.0014 & 0.02586 & 0.0313 \\
\hline \multirow[t]{2}{*}{ ELD } & $\alpha$ & 3.7245 & 0.5294 & 2.68688 & 4.7621 \\
\hline & $\theta$ & 0.0498 & 0.0030 & 0.04392 & 0.0557 \\
\hline $\mathrm{ED}$ & $\theta$ & 0.0145 & 0.0010 & 0.01254 & 0.0165 \\
\hline \multirow[t]{2}{*}{ EED } & $\alpha$ & 8.5952 & 1.3112 & 6.02525 & 11.1652 \\
\hline & $\theta$ & 0.0407 & 0.0027 & 0.03541 & 0.0460 \\
\hline
\end{tabular}


The values reported in Table 3 are the estimated parameters of the distributions, their standard errors and confidence intervals for data set 2 . Table 4 gives the Log-likelihood values, K-S statistic and their p-values, AIC and BIC values for data set 2 .

The results reported in Table 1 and Table 3 reveal that, on the average, the values of AIC, BIC and K-S are smaller for the BEI distribution than the other distributions while the values of log-likelihood (LL) and p-values are higher for the BEI than the other distributions. Hence, the BEI distribution outperforms the other distributions with respect to data set one and two. In addition, a close look at Figure 3 and Figure 4 indicates that BEI fits the data sets better than others. In addition, a close look at Figures 3-6 indicates that BEI fits the data sets better than other competing distributions.

Table 2. The results of the log-lik, AIC, BIC, KS statistic and p-value of the fitted distributions.

\begin{tabular}{cccccc}
\hline Disdtribution & Log-lik & KS Statistic & $P$-value & AIC & BIC \\
\hline BEID & -949.4892 & 0.0523 & 0.6188 & 1906.9780 & 1920.2120 \\
ID & -976.0187 & 0.1369 & 0.0009 & 1954.0370 & 1957.3460 \\
EID & -1024.0920 & 0.2183 & 0.0000 & 2052.1840 & 2058.8000 \\
AD & -976.1313 & 0.1373 & 0.0009 & 1954.2630 & 1957.5710 \\
EAD & -960.5972 & 0.2638 & 0.0000 & 1925.1940 & 1931.8110 \\
LD & -1001.7430 & 0.2154 & 0.0000 & 2005.4860 & 2008.7950 \\
ELD & -959.5830 & 0.0878 & 0.0836 & 1923.1660 & 1929.7830 \\
ED & -1057.3530 & 0.3458 & 0.0000 & 2116.7070 & 2120.0150 \\
EED & -958.0064 & 0.6986 & 0.0000 & 1920.0130 & 1926.6290 \\
\hline
\end{tabular}

Table 3. The MLEs of the parameters of the fitted distributions and their confidence intervals.

\begin{tabular}{cccccc}
\hline Distribution & Parameter & MLE & Standard Error & Lower Bound & Upper Bound \\
\hline \multirow{2}{*}{ BEID } & $\alpha$ & 0.0578 & 0.1979 & -0.3300 & 0.4460 \\
& $\theta$ & 0.3312 & 0.2401 & -0.1390 & 0.8020 \\
& $a$ & 54.1596 & 190.3422 & -318.9110 & 427.2300 \\
ID & $b$ & 3.8958 & 4.2409 & -4.4160 & 12.2080 \\
EID & $\theta$ & 0.4607 & 0.0403 & 0.3820 & 0.5400 \\
& $\alpha$ & 0.6755 & 0.0980 & 0.4830 & 0.8680 \\
AD & $\theta$ & 0.4072 & 0.0518 & 0.3060 & 0.5090 \\
EAD & $\theta$ & 0.4504 & 0.0401 & 0.3720 & 0.5290 \\
& $\alpha$ & 3.8552 & 1.1794 & 1.5440 & 6.1670 \\
LD & $\theta$ & 0.7116 & 0.0745 & 0.5660 & 0.8580 \\
ELD & $\theta$ & 0.2845 & 0.0322 & 0.2210 & 0.3480 \\
& $\alpha$ & 6.0208 & 1.8280 & 2.4380 & 9.6040 \\
ED & $\theta$ & 0.5664 & 0.0643 & 0.4400 & 0.6920 \\
EED & $\theta$ & 0.1599 & 0.0253 & 0.1100 & 0.2090 \\
& $\alpha$ & 9.5126 & 2.8956 & 3.837 & 15.188 \\
& $\theta$ & 0.4498 & 0.0578 & 0.337 & 0.563 \\
\hline
\end{tabular}




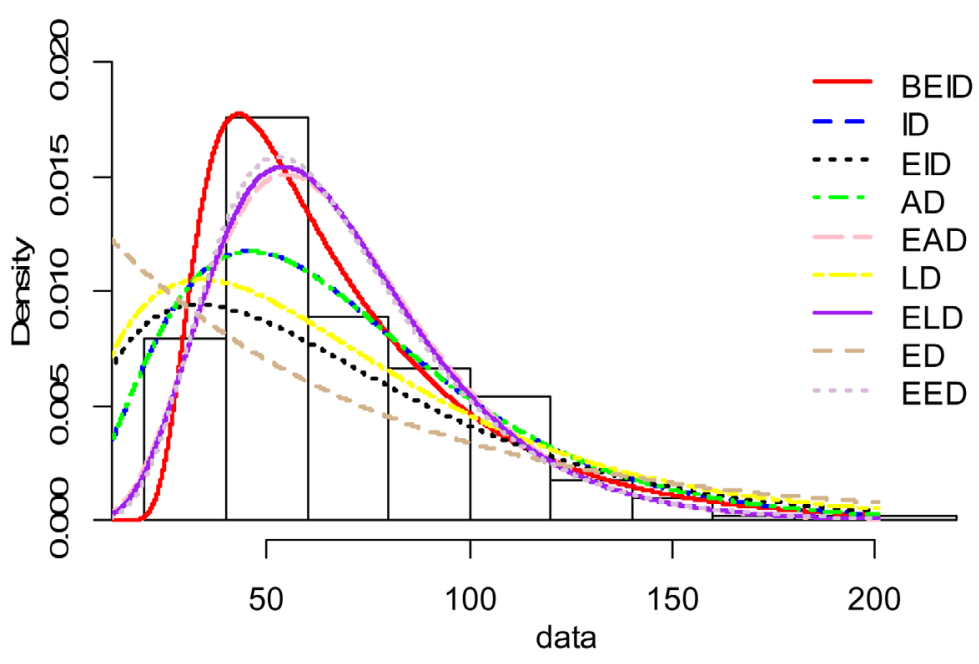

Figure 3. The histogram and PDFs of fitted models for data set one.

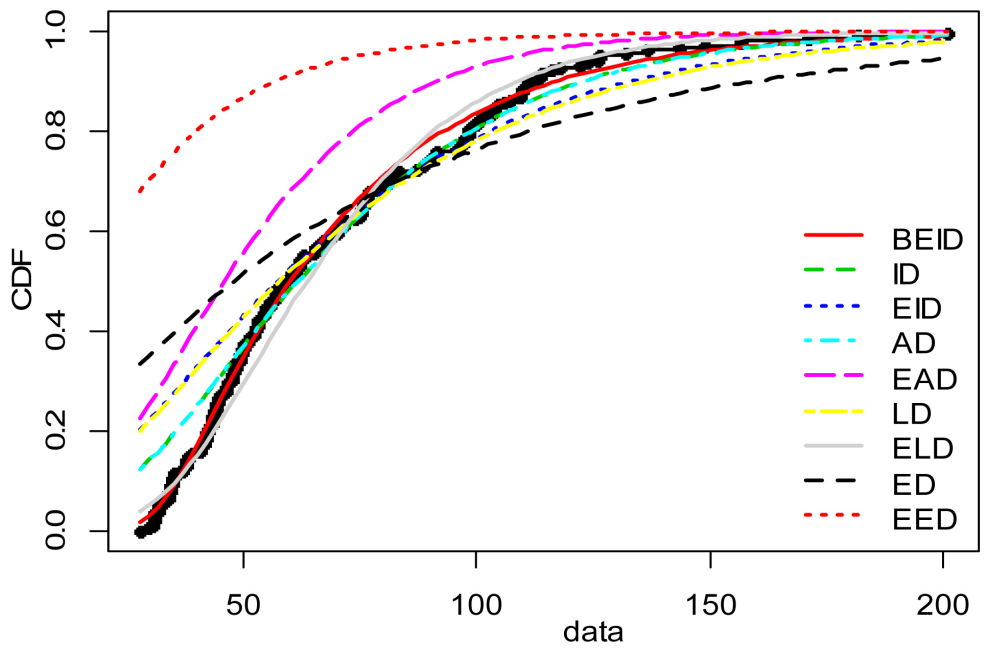

Figure 4. CDFs of fitted models for data set one.

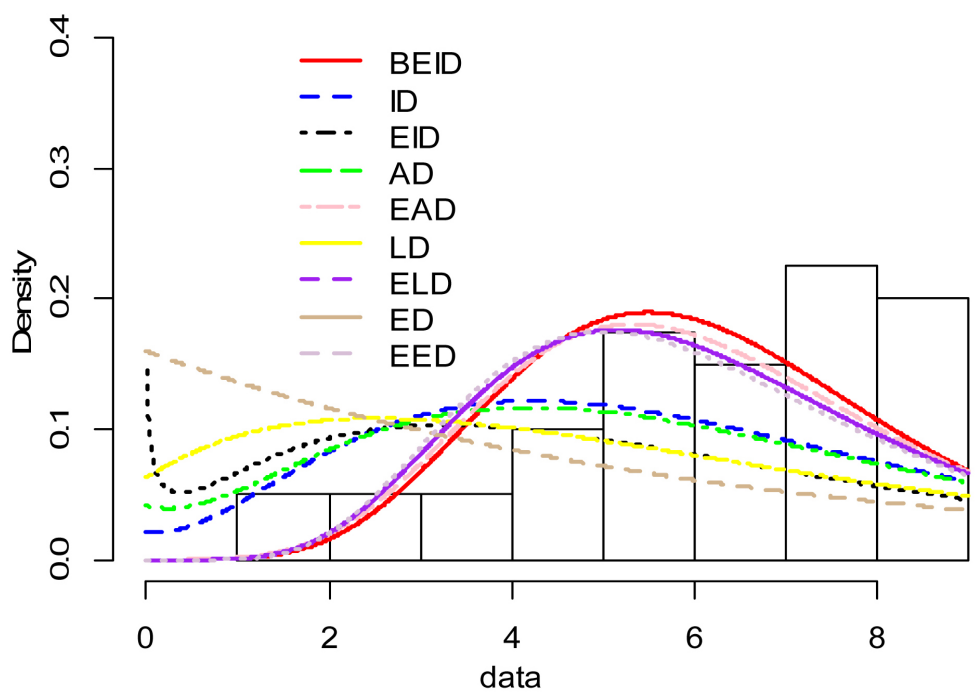

Figure 5. The histogram and PDFs of fitted models for data set two. 


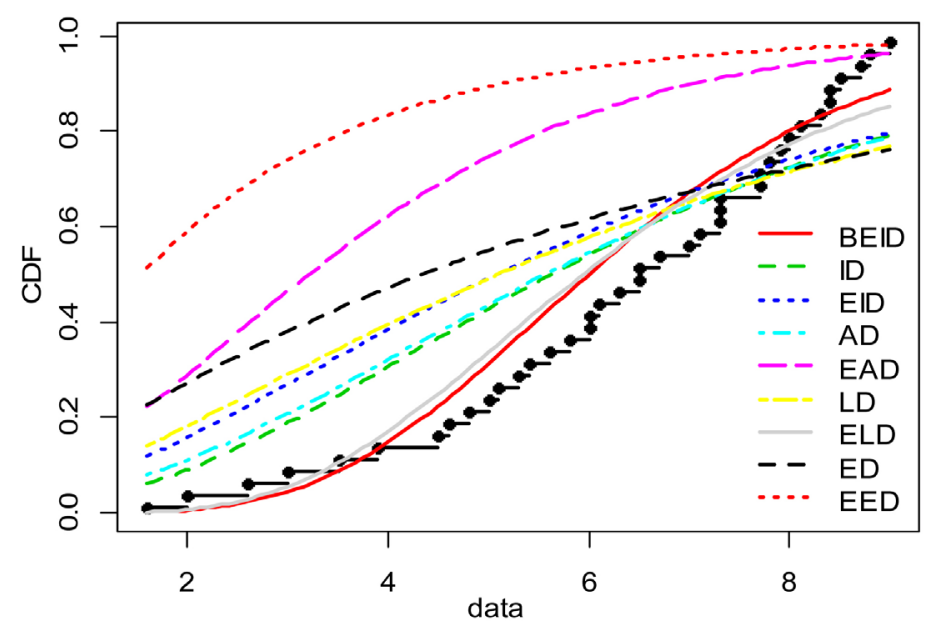

Figure 6. CDFs of fitted models for data set two.

Table 4. The results of the log-lik, AIC, BIC, KS statistic and p-value of the fitted distributions.

\begin{tabular}{cccccc}
\hline Disdtribution & Log-lik & KS Statistic & $P$-value & AIC & BIC \\
\hline BEID & -87.0505 & 0.1256 & 0.5134 & 182.1010 & 188.8565 \\
ID & -95.6793 & 0.2178 & 0.0380 & 193.3586 & 195.0475 \\
EID & -105.2049 & 0.2899 & 0.0018 & 214.4099 & 217.7877 \\
AD & -96.8592 & 0.2300 & 0.0242 & 195.7185 & 197.4073 \\
EAD & -88.1004 & 0.5390 & 0.0000 & 180.2009 & 183.5786 \\
LD & -104.2854 & 0.2949 & 0.0014 & 210.5708 & 212.2597 \\
ELD & -89.1819 & 0.1467 & 0.3234 & 182.3639 & 185.7416 \\
ED & -113.3193 & 0.3631 & 0.0000 & 230.3274 & 230.3274 \\
EED & -90.1427 & 0.7179 & 0.0000 & 184.2853 & 187.6631 \\
\hline
\end{tabular}

\section{Concluding Remarks}

In this article, we propose a new distribution called the Beta-Exponentiated Ishita (BEI) distribution for lifetime analysis. We provide for the new distribution expressions for the distribution function, density function, moments, cumulants, skewness, kurtosis, moment generating function, characteristic function, mean deviations, entropies, Bonferroni and Lorenz curves, stress-strength reliability, distribution of order statistics and its moments. Estimation of the parameters by maximum likelihood method is discussed. Two applications to real lifetime data show that the BEI distribution is more flexible than its competitors and can be used quite effectively in analysing positively skewed and heavy tailed data.

\section{Conflicts of Interest}

The authors declare no conflicts of interest regarding the publication of this paper. 


\section{References}

[1] Shanker, R. and Shukla, K.K. (2017) Ishita Distribution and Its Applications. Biometrics \& Biostatistics International Journal, 5, 1-9. https://doi.org/10.15406/bbij.2017.05.00126

[2] Al-Nasser, A.D., Al-Omari, A.I., Bani-Mustafa, A. and Jaber, K. (2018) Developing Single-Acceptance Sampling Plans Based on a Truncated Lifetime Test for an Ishita Distribution. Statistics in Transition New Series, 19, 393-406. https://doi.org/10.21307/stattrans-2018-022

[3] Shukla, K.K. and Shanker, R. (2018) Power Ishita Distribution and Its Application to Model Lifetime Data. Statistics in Transition New Series, 19, 135-148. https://doi.org/10.21307/stattrans-2018-008

[4] Al-Omari, A.I., Al-Nasser, A.D. and Ciavolino, E.A. (2019) A Sized-Biased Ishita Distribution and Application to Real Data. Quality \& Quantity, 53, 493-512. https://doi.org/10.1007/s11135-018-0765-y

[5] Hassan, A., Dar, S.A. and Ahmad, P.B. (2019) Poisson Ishita Distribution: A New Compounding Probability Model. Int. Organ. Sci. Res. J. Eng., 9, 38-46.

[6] Gharaibeh, M.M. and Al-Omari, A.I. (2019) Transmuted Ishita Distribution and Its Applications. Journal of Statistics Applications \& Probability, 8, 67-81. https://doi.org/10.18576/jsap/080201

[7] Rather, A.A. and Subramanian, C. (2019) Exponentiated Ishita Distribution: Properties and Applications. International Journal of Management, Technology and Engineering, 9, 2473-2484.

[8] Eugene, N., Lee, C. and Famoye, F. (2002) Beta-Normal Distribution and Its Applications. Communications in Statistics. Theory and Methods, 31, 497-512. https://doi.org/10.1081/STA-120003130

[9] Nadarajah, S. and Kotz, S. (2004) The Beta Gumbel Distribution. Mathematical Problems in Engineering, 4, 323-332. https://doi.org/10.1155/S1024123X04403068

[10] Nadarajah, S. and Gupta, A.K. (2004) The Beta-Frechet Distribution. Far East Journal of Theoretical Statistics, 14, 15-24.

[11] Famoye, F., Lee, C. and Olumolade, O. (2005) The Beta-Weibull Distribution. Journal of Statistical Theory and Applications, 4, 121-136.

[12] Nadarajah, S. and Kotz, S. (2006) The Beta Exponential Distribution. Reliability Engineering and System Safety, 91, 689-697. https://doi.org/10.1016/j.ress.2005.05.008

[13] Gupta, A.K. and Nadarajah, S. (2006) Beta Bessel Distribution. International Journal of Mathematics and Mathematical Sciences, 2006, Article ID: 16156. https://doi.org/10.1155/IJMMS/2006/16156

[14] Siddiqui, S.A., Dwivedi, S., Dwivedi, P. and Alam, M. (2016) Beta Exponentiated Mukherjii Islam Distribution, Mathematical Study of Different Properties. Global Journal of Pure and Applied Mathematics, 12, 951-964.

[15] Kong, L., Lee, C. and Sepanski, J.H. (2007) On the Properties of Beta-Gamma Distribution. Journal of Modern Applied Statistical Methods, 6, 187-211. https://doi.org/10.22237/jmasm/1177993020

[16] Akinsete, A., Famoye, F. and Lee, C. (2008) The Beta-Pareto Distribution. Statistics, 42, 547-563. https://doi.org/10.1080/02331880801983876

[17] Akinsete, A. and Lowe, C. (2009) Beta-Rayleigh Distribution in Reliability Measure, Section on Physical and Engineering Sciences. Proceedings of the American Statistical Association, Vol. 1, 3103-3107. 
[18] Amusan, G.E. (2010) The Beta Maxwell Distribution. Marsh. Digit. Schol. Stat., 16, 409-430.

[19] Souza, W.B., Santos, A.H. and Cordeiro, G.M. (2010) The Beta Generalized Exponential Distribution. Journal of Statistical Computation and Simulation, 80, 159-172. https://doi.org/10.1080/00949650802552402

[20] Cordeiro, G.M. and Lemonte, A.J. (2011a) The Beta Laplace Distribution. Statistics \& Probability Letters, 81, 973-982. https://doi.org/10.1016/j.spl.2011.01.017

[21] Cordeiro, G.M. and Lemonte, A.J. (2011b) The Beta-Birnbaum-Saunders Distribution: An Improved Distribution for Fatigue Life Modelling. Computational Statistics \& Data Analysis, 55, 1445-1461. https://doi.org/10.1016/j.csda.2010.10.007

[22] Castellares, F., Montenegro, L.C. and Cordeiro, G.M. (2011) The Beta Lognormal Distribution. Journal of Statistical Computation and Simulation, 83, 203-228. https://doi.org/10.1080/00949655.2011.599809

[23] Alshawarbeh, E., Lee, C. and Famoye, F. (2012) The Beta-Cauchy Distribution. Journal of Probability and Statistical Science, 10, 41-57.

[24] Shittu, O.I. and Adepoju, K.A. (2012) On the Beta-Nakagami Distribution. Progress in Applied Mathematics, 5, 49-58.

[25] Cordeiro, G.M., Nobre, J.S., Pescim, R.R. and Ortega, E.M.M. (2012) The Beta Moyal: A Useful-Skew Distribution. International Journal of Research and Reviews in Applied Sciences, 10, 171-192.

[26] Condino, F. and Domma, F. (2013) The Beta-Dagum Distribution. Communications in Statistics-Theory and Methods, 42, 4070-4090.

https://doi.org/10.1080/03610926.2011.647219

[27] Cordeiro, G.M., Gomes, A.E., Da-Silva, C.Q. and Ortega, E.M.M. (2013) The Beta Exponentiated Weibull Distribution. Statistical Papers, 83, 114-138.

https://doi.org/10.1080/00949655.2011.615838

[28] Gomes, A.E., Da-Silva, C.Q., Cordeiro, G.M. and Ortega, E.M.M. (2013) The Beta Burr III Model for Lifetime Data. Brazilian Journal of Probability and Statistics, 27, 502-543. https://doi.org/10.1214/11-BJPS179

[29] Jafari, A.A., Tahmasebi, S. and Alizadeh, M. (2014) The Beta-Gompertz Distribution. Revista Colombiana de Estadstica, 37, 141-158. https://doi.org/10.15446/rce.v37n1.44363

[30] Lemonte, A.J. (2014) The Beta Log-Logistic Distribution. Brazilian Journal of Probability and Statistics, 28, 313-332. https://doi.org/10.1214/12-BJPS209

[31] Rodrigues, J.A., Silva, A.P.C.M. and Hamedani, G.G. (2015) The Beta Exponentiated Lindley Distribution. Journal of Statistical Theory and Applications, 14, 60-75.

https://doi.org/10.2991/jsta.2015.14.1.6

[32] Ownuk, J. (2015) The Beta Exponentiated Gumbel Distribution. Journal of the Iranian Statistical Society, 14, 1-14.

[33] MirMostafaee, S.M.T.K., Mahdizadeh, M. and Nadarajah, S. (2015) The Beta Lindley Distribution. Journal of Data Science, 13, 603-626. https://doi.org/10.6339/JDS.201507 13(3).0010

[34] Fischer, M.J. and Vaughan, D. (2016) The Beta-Hyperbolic Secant Distribution. Austrian Journal of Statistics, 39, 245-258. https://doi.org/10.17713/ajs.v39i3.247

[35] Merovci, F., Khalee, M.A., Ibrahim, N.A. and Shitan, M. (2016) The Beta Burr Type X Distribution: Properties with Application. Springer Plus, 5, 1-18.

https://doi.org/10.1186/s40064-016-2271-9 
[36] Mead, M.E., Afify, A.Z., Hamedani, G.G. and Ghosh, I. (2017) The Beta Exponential Frechet Distribution with Applications. Austrian Journal of Statistics, 46, 41-63. https://doi.org/10.17713/ajs.v46i1.144

[37] Dias, C.R., Alizadeh, M. and Cordeiro, G.M. (2018) The Beta Nadarajah-Haghighi Distribution. Hacettepe Journal of Mathematics and Statistics, 47, 1302-1320.

[38] Shahzad, M.N., Ullah, E. and Hussanan, A. (2019) Beta Exponentiated Modified Weibull Distribution: Properties and Application. Symmetry, 11, 781. https://doi.org/10.3390/sym11060781

[39] Bonferroni, C.E. (1930) Elementi di Statistica Generale. Seeber, Firenze.

[40] Renyi, A. (1961) On Measures of Entropy and Information. Proceedings of the 4th Berkeley Symposium on Mathematical Statistics and Probability, Vol. 1, 547-561.

[41] Lorenz, M.O. (1905) Methods of Measuring the Concentration of Wealth. Publications-American Statistical Association, 9, 209-219.

https://doi.org/10.2307/2276207

[42] Tsallis, C. (1988) Possible Generalization of Boltzmann-Gibbs Statistics. Journal of Statistical Physics, 52, 479-487. https://doi.org/10.1007/BF01016429

[43] Lehmann, L.E. and Casella, G. (1998) Theory of Point Estimation. 2nd Edition, Springer, New York.

[44] Weisberg, S. (2005) Applied Linear Regression. 3rd Edition, Wiley and Sons, Inc., New York. https://doi.org/10.1002/0471704091

[45] Xu, K., Xie, M., Tang, L.C. and Ho, S.L. (2003) Application of Neural Networks in Forecasting Engine Systems Reliability. Applied Soft Computing, 2, 255-268.

https://doi.org/10.1016/S1568-4946(02)00059-5 